\title{
On the embeddability of certain infinitely divisible probability measures on Lie groups
}

\author{
S.G. Dani, Yves Guivarc'h and Riddhi Shah
}

June 1, 2018

\begin{abstract}
We describe certain sufficient conditions for an infinitely divisible probability measure on a class of connected Lie groups to be embeddable in continuous one-parameter semigroups of probability measures. (Theorem 1.3). This enables us in particular to conclude the embeddability of all infinitely divisible probability measures on certain Lie groups, including the so called Walnut group (Corollary 1.5). The embeddability is concluded also under certain other conditions (Corollary 1.4 and Theorem 1.6).
\end{abstract}

\section{Introduction}

Let $G$ be a Lie group. We denote by $P(G)$ the space of probability measures on $G$, equipped with the convolution product and the usual weak* topology. A $\mu \in P(G)$ is said to be infinitely divisible if it admits convolution roots of all orders and it is said to be embeddable if there exists a continuous one-parameter convolution semigroup $\left\{\mu_{t}\right\}_{t>0}$ such that $\mu_{1}=\mu$. It has been conjectured that when $G$ is a connected Lie group every infinitely divisible probability measure on $G$ is embeddable; the latter is known as the embedding property. The results in 8 and [9] show that this holds under the additional condition that the nilradical of $G$ contains no torus of positive dimension. The embedding property is also known to hold for all nilpotent Lie groups. An example of a nonnilpotent Lie group for which the above condition fails occurs in dimension 4. Partial results towards the embedding property were proved for this group, termed as the Walnut group, in [10], taking off from the Ph.D. thesis of S. Walker.

In this paper we prove some new results on the embeddability of infinitely divisible measures on a class of connected Lie groups, under an assumption on the measure (see Theorem 1.3). This in particular enables us to settle the em- 
bedding problem for the Walnut group, and also a larger group containing it (see Corollary [1.5).

We now begin with some more notation and a formal statement of the main results. Let $G$ be a Lie group. For any closed subset $S$ of $G$ we denote by $P(S)$ the subspace of $P(G)$ consisting of the probability measures whose support is contained in $S$. For a subset $\Lambda$ of probability measures on $G$ we denote by $G(\Lambda)$ the smallest closed subgroup of $G$ containing the supports of all $\lambda \in \Lambda$, and by $Z(\Lambda)$ the centraliser of $G(\Lambda)$ in $G$. For $\lambda \in P(G), G(\{\lambda\})$ and $Z(\{\lambda\})$ will be written as $G(\lambda)$ and $Z(\lambda)$ respectively. For $\lambda \in P(G)$ we denote by $N(\lambda)$ the normaliser of $G(\lambda)$ in $G$. We recall that given a (convolution) root $\rho$ of $\lambda$, there exists $x \in N(\lambda)$ such that $\rho \in P(x G(\lambda))$; furthermore, if $\rho^{n}=\lambda$ then for every such $x$ we have $x^{n} \in G(\lambda)$ (see, for instance, [11], Lemma 2.2).

We shall mainly be concerned with Lie groups which admit surjective homomorphisms onto almost algebraic groups, with kernels contained in the center; see Theorem 1.3 below. By an almost algebraic group we mean a Lie group which is (Lie isomorphic to) a subgroup of finite index in a real algebraic group (the group of real points of an algebraic group defined over $\mathbb{R}$ ); see $[8], \S 2$, for some generalities in this respect. Let $\widetilde{G}$ be an almost algebraic group. For a subset $\Lambda$ of $P(\widetilde{G})$ we shall denote by $\widetilde{G}(\Lambda)$ the smallest almost algebraic subgroup of $\widetilde{G}$ containing $G(\Lambda)$ (or, equivalently, supp $\lambda$ for all $\lambda \in \Lambda$ ). For $\nu \in P(\widetilde{G}), \widetilde{G}(\nu)$ stands for $\widetilde{G}(\{\nu\})$ and $\widetilde{N}(\nu)$ denotes the normaliser of $\widetilde{G}(\nu)$ in $\widetilde{G}$. We note that for any $\nu \in P(\widetilde{G}), Z(\nu)$ is an algebraic subgroup of $\widetilde{G}$ and it is also the centraliser of $\widetilde{G}(\nu)$. Also, for any $\nu \in P(\widetilde{G}), N(\nu)$ is contained in $\widetilde{N}(\nu)$.

For a Lie subgroup $H$ we denote by $H^{0}$ the connected component of the identity in $H$; (in the case of $G(\lambda), Z(\lambda)$ etc. the identity component will be written as $G^{0}(\lambda), Z^{0}(\lambda)$ etc. $)$.

Definition 1.1. Let $\mu \in P(G)$. A subset $\Psi$ consisting of roots of $\mu$ in $P(G)$ is called a root cluster of $\mu$ if the following conditions are satisfied:

i) if $n \in \mathbb{N}$ and $\rho \in \Psi$ is a $n$th root of $\mu$ then $\rho^{k} \in \Psi$ for all $k$ dividing $n$;

ii) for all $\rho \in \Psi$ and $z \in Z(\mu), z \rho z^{-1} \in \Psi$;

iii) for all $n \in \mathbb{N},\left\{\rho \in \Psi \mid \rho^{n}=\mu\right\}$ is a nonempty closed subset of $P(G)$.

We note that if $\mu$ is infinitely divisible then the set of all roots of $\mu$ in $P(G)$ is a root cluster for $P(G)$.

Definition 1.2. Let $\mu \in P(G)$. A family $\left\{\mu_{r}\right\}_{r \in \mathbb{Q}^{+}}$, where $\mathbb{Q}^{+}$is the additive semigroup consisting of all positive rationals, is called a rational embedding of $\mu$ if $\mu_{1}=\mu$ and $r \mapsto \mu_{r}$ is a homomorphism of $\mathbb{Q}^{+}$into $P(G)$.

It is known that every rational embedding $\left\{\mu_{r}\right\}_{r \in \mathbb{Q}^{+}}$extends uniquely to an 
embedding $\left\{\mu_{t}\right\}_{t \geq 0}$ (see [6]), so if $\mu$ admits a rational embedding then it is continuously embeddable.

We prove the following.

Theorem 1.3. Let $G$ be a connected Lie group admitting a surjective continuous homomorphism $p: G \rightarrow \widetilde{G}$ onto an almost algebraic group $\widetilde{G}$, such that ker $p$ is contained in the center of $G$ and $(\operatorname{ker} p)^{0}$ is compact. Let $T=(\operatorname{ker} p)^{0}$ and $q: G \rightarrow G / T$ be the quotient homomorphism. Let $\mu \in P(G)$ be such that $q(\mu)$ has no nontrivial idempotent factor in $P(G / T)$. Let $\Psi$ be a root cluster of $\mu$. Then there exists a rational embedding $\left\{\mu_{r}\right\}_{r \in \mathbb{Q}^{+}}$of $\mu$ such that $\mu_{r} \in \Psi$ for all $r \in \mathbb{Q}^{+}$; in particular, if $\mu$ is infinitely divisible then it is embeddable.

From the theorem we deduce also the following corollary, for a general connected Lie group.

Corollary 1.4. Let $G$ be a connected Lie group and $N$ be the nilradical of $G$. If $\mu \in P(N)$ is infinitely divisible in $P(G)$ then $\mu$ is embeddable in $P(G)$.

We next describe certain special groups for which the embedding problem can now be settled. Let $H$ be the 3-dimensional Heisenberg group, $Z$ be the (onedimensional) center of $H$ and $D$ an infinite cyclic subgroup of $Z$. Let $N=H / D$ and $T=Z / D$. Then $N$ is a nilpotent Lie group and $T$ is the maximal torus in $N$. The group $S L(2, \mathbb{R})$ has a canonical action on $H$ as a group of automorphisms, such that $Z$ is point-wise fixed and the quotient action on $N / Z$, which we view as $\mathbb{R}^{2}$, is the usual linear action of $S L(2, \mathbb{R})$ on $\mathbb{R}^{2}$. As $D$ is pointwise fixed under the action we get a quotient action of $S L(2, \mathbb{R})$ on $N$, as a group of automorphisms. Let $G$ be the semidirect product of $S L(2, \mathbb{R})$ and $N$ with respect to the action. It may be recalled here that this group plays an important role in the study of Jacobi forms; see [12]. We view $S L(2, \mathbb{R})$ and $N$ as subgroups of $G$, canonically. Let $K$ be the subgroup of $S L(2, \mathbb{R})$ consisting of rotations of $\mathbb{R}^{2}$ around 0 (with respect to a fixed Hilbert norm on $\mathbb{R}^{2}$ ). The subgroup of $G$ containing $K$ and $N$, which is the semidirect product of the two subgroups with respect to the $K$ action on $N$, is the Walnut group; see [10] and [15] for more details. We shall denote the subgroup by $W$.

Applying Theorem 1.3 together with certain known results we deduce the following.

Corollary 1.5. Let $G$ be the semidirect product of $S L(2, \mathbb{R})$ and $N$ as above. Let $H$ be a closed connected subgroup of $G$. Then every infinitely divisible probability measure in $P(H)$ is embeddable in $P(H)$. In particular, every probability measure which is infinitely divisible in $P(W)$ is embeddable in $P(W)$.

In the proof of Theorem 1.3 the condition that $q(\mu)$ has no idempotent factor is needed only in the case $G(\mu) / \overline{[G(\mu), G(\mu)]}$ is not compact. Using this observation 
together with some facts about Lie groups we conclude also the following result, for all connected Lie groups (not involving the condition as in Theorem 1.3).

Theorem 1.6. Let $G$ be a connected Lie group and $\mu \in P(G)$ be infinitely divisible. Suppose that $G(\mu) / \overline{[G(\mu), G(\mu)]}$ is compact. Then $\mu$ is embeddable.

\section{Some generalities on central extensions}

Let $G$ be a Lie group, not necessarily connected, and suppose that there exist an almost algebraic group $\widetilde{G}$ and a surjective continuous homomorphism $p: G \rightarrow \widetilde{G}$ such that ker $p$ is a compactly generated subgroup contained in the center of $G$ and $(\operatorname{ker} p)^{0}$ is compact. Then ker $p$ has a unique maximal compact subgroup which we shall denote by $A$, and ker $p$ is isomorphic to $A \times \mathbb{Z}^{r}$ for some $r \geq 0$. The connected component of the identity in $A$, which is a torus, will be denoted by $T$.

Let $\mu \in P(G)$ and $\nu=p(\mu)$. For each $z \in Z(\nu)$ we define a map $\psi_{z}: \widetilde{G}(\nu) \rightarrow$ ker $p$ as follows: let $x \in \widetilde{G}(\nu)$, and $\xi, \zeta \in G$ be such that $p(\xi)=x$ and $p(\zeta)=z$ (we recall that $p$ is surjective); since $x$ and $z$ commute with each other, the element $\zeta \xi \zeta^{-1} \xi^{-1}$ is contained in $\operatorname{ker} p$, and since $\operatorname{ker} p$ is central in $G$ it follows that this element is independent of the choices of $\xi$ and $\zeta$ (depends only on $x$ and $z$ ); we define $\psi_{z}(x)$ to be $\zeta \xi \zeta^{-1} \xi^{-1}$. Clearly $\psi_{z}$ is a continuous map for all $z$. Also, for $z=p(\zeta) \in Z(\nu)$, given $x=p(\xi), x^{\prime}=p\left(\xi^{\prime}\right) \in \widetilde{G}(\nu)$, we have $\psi_{z}\left(x x^{\prime}\right)=$ $\zeta \xi \xi^{\prime} \zeta^{-1}\left(\xi \xi^{\prime}\right)^{-1}=\zeta \xi \zeta^{-1} \psi_{z}\left(x^{\prime}\right) \xi^{-1}=\psi_{z}(x) \psi_{z}\left(x^{\prime}\right)$, as $\psi_{z}\left(x^{\prime}\right)$ is central. This shows that each $\psi_{z}$ is a homomorphism. Being an almost algebraic group $\widetilde{G}(\nu)$ has only finitely many connected components, and hence it follows also that for all $z \in Z(\nu)$ the image of $\psi_{z}$ is contained in $A$. Furthermore, as the image is abelian, $\psi_{z}$ factors canonically to a homomorphism of $\widetilde{G}(\nu) /[\widetilde{G}(\nu), \widetilde{G}(\nu)]$ into $A$ (we note that as $\widetilde{G}(\nu)$ is almost algebraic, $[\widetilde{G}(\nu), \widetilde{G}(\nu)]$ is also almost algebraic and hence in particular a closed subgroup); we shall denote this homomorphism also by $\psi_{z}$.

Lemma 2.1. Let $Q$ be the smallest closed normal subgroup of $\widetilde{G}(\nu)$ such that $\widetilde{G}(\nu) / Q$ is a vector group, and let $\widetilde{V}=\widetilde{G}(\nu) / Q$. Let $K$ be the smallest closed normal subgroup of $Z^{0}(\nu)$ such that $Z^{0}(\nu) / K$ is a vector group. Then we have the following.

i) for all $z \in Z^{0}(\nu), \psi_{z}$ factors to a continuous homomorphism of $\widetilde{V}$ into $T$;

ii) for all $z \in K, \psi_{z}$ is trivial, that is $\psi_{z}(x)=e$ for all $x \in \widetilde{G}(\nu)$.

Proof. Let Hom $(\widetilde{G}(\nu) /[\widetilde{G}(\nu), \widetilde{G}(\nu)], A)$ be the group of continuous homomorphisms of $\widetilde{G}(\nu) /[\widetilde{G}(\nu), \widetilde{G}(\nu)]$ into $A$, under pointwise multiplication, equipped with the compact open topology. Let $\psi: Z(\nu) \rightarrow \operatorname{Hom}(\widetilde{G}(\nu) /[\widetilde{G}(\nu), \widetilde{G}(\nu)], A)$ be the map 
$z \mapsto \psi_{z}$ for all $z \in \widetilde{Z}(\nu)$ defined as above. It can be seen that $\psi$ is a continuous homomorphism.

As $\widetilde{G}(\nu)$ is an almost algebraic group, $\widetilde{G}(\nu) /[\widetilde{G}(\nu), \widetilde{G}(\nu)]$ is an abelian Lie group with finitely many connected components. By Pontryagin duality theory this implies that Hom $(\widetilde{G}(\nu) /[\widetilde{G}(\nu), \widetilde{G}(\nu)], A)$ has no compact subgroup of positive dimension. In particular its connected component of the identity is a vector group, (namely a topological group isomorphic to $\mathbb{R}^{n}$ for some $n \geq 0$ ). The identity component therefore consists of homomorphisms that factor to $\widetilde{V}$ and have their image contained in $T$; we shall denote the latter subgroup by $\operatorname{Hom}(\widetilde{V}, T)$. Then by continuity, $\psi\left(Z^{0}(\nu)\right)$ is contained in $\operatorname{Hom}(\widetilde{V}, T)$; that is, for all $z \in Z^{0}(\nu), \psi_{z}$ factors to a continuous homomorphism of $\widetilde{V}$ into $T$. This proves (i). Assertion (ii) is immediate from the definition of $K$ and the fact that $\psi\left(Z^{0}(\nu)\right)$ is a vector group. This proves the Lemma.

Remark 2.2. If $\zeta \in p^{-1}(Z(\nu))$ is such that $\psi_{p(\zeta)}$ is trivial then $\zeta \in Z(\mu)$. Indeed, for any $\xi \in G(\mu)$ we have $\zeta \xi \zeta^{-1} \xi^{-1}=\psi_{p(\zeta)}(p(\xi))=e$. Lemma 2.1 implies that $(z, x) \mapsto \psi(z, x)$ is a continuous bi-homomorphism of $\widetilde{V} \times Z^{0}(\nu) / K$ into $T$. Using Pontryagin duality we see that this map defines a bilinear pairing into the universal cover of $T$.

Let $K$ be the subgroup as in Lemma 2.1. We note that $K$ is the subgroup containing $\left[Z^{0}(\nu), Z^{0}(\nu)\right]$ (which is an almost algebraic normal subgroup of $Z^{0}(\nu)$ ) and such that $K /\left[Z^{0}(\nu), Z^{0}(\nu)\right]$ is the maximal torus in the abelian Lie group $Z^{0}(\nu) /\left[Z^{0}(\nu), Z^{0}(\nu)\right]$. Let $W$ denote the vector space $Z^{0}(\nu) / K$. For $x \in \tilde{N}(\nu)$ let $\tau_{x}: W \rightarrow W$ be the linear transformation of $W$ induced by the conjugation action of $x$ on $Z^{0}(\nu)$, and let $W_{x}$ be the range of $\tau_{x}-I$, where $I$ denotes the identity transformation of $W$. We note that $W_{x}$ is the smallest $\tau_{x}$-invariant subspace such that the factor of $\tau_{x}$ on $W / W_{x}$ is the identity automorphism. We shall denote by $K(\nu, x)$ the subgroup of $Z^{0}(\nu)$ containing $K$ and such that $W_{x}=K(\nu, x) / K$.

Lemma 2.3. Let $x \in \tilde{N}(\nu)$ and $\left\{g_{t}\right\}$ be a one-parameter subgroup in $\widetilde{G}(\nu)$ such that $x g_{t} x^{-1} g_{t}^{-1}$ is contained in $Q$, namely $\left\{g_{t} Q\right\}$ which is a one-dimensional subspace of $\widetilde{V}=\widetilde{G}(\nu) / Q$ is invariant under the action of $x$ on $\widetilde{V}$ induced by the conjugation action. Then $\psi_{z}\left(g_{t}\right)=e$ for all $t \in \mathbb{R}$ and $z \in K(\nu, x)$.

Proof. Let $\mathfrak{G}$ denote the Lie algebra of $G$. Let $\xi \in \mathfrak{G}$ be such that $p(\exp t \xi)=g_{t}$ for all $t \in \mathbb{R}$ and let $\zeta \in \mathfrak{G}$ be any element such that $\{p(\exp t \zeta)\}$ is contained in $Z^{0}(\nu)$. Then we have $\operatorname{Ad} x([\xi, \zeta])=[\operatorname{Ad} x(\xi), \operatorname{Ad} x(\zeta)]=[\xi+\theta, \operatorname{Ad} x(\zeta)]$, where $\theta$ is an element of the Lie subalgebra of $p^{-1}(Q)$. Since the restriction of $\psi_{z}$ to $Q$ is trivial for all $z \in Z^{0}(\nu)$ (by Lemma 2.1) it follows that $[\theta, \operatorname{Ad} x(\zeta)]=0$, and hence we have $\operatorname{Ad} x([\xi, \zeta])=[\xi, \operatorname{Ad} x(\zeta)]$. On the other hand since $[\xi, \zeta]$ belongs to the Lie subalgebra corresponding to $T$, which is contained in the center of 
$G$, we have $\operatorname{Ad} x([\xi, \zeta])=[\xi, \zeta]$. Therefore $[\xi, \operatorname{Ad} x(\zeta)]=[\xi, \zeta]$, or equivalently $[\xi, \operatorname{Ad} x(\zeta)-\zeta]=0$ for all $\zeta$ as above. This shows that for all $t \in \mathbb{R}$, $\exp t \xi$ centralises $K(\nu, x)$. Equivalently $\psi_{z}\left(g_{t}\right)=e$ for all $t \in \mathbb{R}$ and $z \in K(\nu, x)$.

We need the following consequence of the lemma in the proof of Theorem 1.3.

Corollary 2.4. Let $\Phi$ be a set of roots of $\nu$. Let $M$ be the smallest closed normal $\widetilde{G}(\Phi)$-invariant subgroup of $Z^{0}(\nu)$ such that $Z^{0}(\nu) / M$ is a vector group and the induced $\widetilde{G}(\Phi)$-action on $Z^{0}(\nu) / M$ is trivial. Let $g \in \widetilde{G}(\nu)$ be such that $g Q$ is a fixed point of the $\widetilde{G}(\Phi)$-action. Then $\psi_{z}(g)=$ e for all $z \in M$.

Proof. Since $\widetilde{G}(\nu) / Q$ is a vector group, the subspace spanned by $g Q$ is pointwise fixed under the $\widetilde{G}(\Phi)$-action and hence there exists a one-parameter subgroup $\left\{g_{t}\right\}$ in $\widetilde{G}(\nu)$ such that $g_{1}=g$ and $g_{t} Q$ is pointwise fixed by $\widetilde{G}(\Phi)$ for all $t \in \mathbb{R}$. Then for any $x \in \widetilde{G}(\Phi)$, by Lemma 2.3 we get that $\psi_{z}\left(g_{t}\right)=e$ for all $t \in \mathbb{R}$ and $z \in K(\nu, x)$, in the notation as above. Let $W^{\prime}$ be the subspace spanned by all $W_{x}, x \in \widetilde{G}(\Phi)$. Then the preceding conclusion (together with the definition of $W_{x}$ ) implies that $\psi_{z}(g)=e$ for all $z \in Z^{0}(\nu)$ such that $z K \in W^{\prime}$. Now, $W^{\prime}$ is invariant under the $\widetilde{G}(\Phi)$ action, and since $W_{x}$ is contained in $W^{\prime}$ for all $x \in \widetilde{G}(\Phi)$ it follows that the $\widetilde{G}(\Phi)$-action on the quotient $W / W^{\prime}$ is trivial. Hence $M$ as in the hypothesis is contained in $W^{\prime}$. This proves the corollary.

\section{Measures on vector spaces}

In this section we note certain properties of measures on vector spaces. For an abelian Lie group $H$ we denote by $\widehat{H}$ its dual group and for a finite measure $\lambda$ on $H$ we denote by $\widehat{\lambda}$ the Fourier transform of $\lambda$ defined on $\widehat{H}$. A function $f$ on $\widehat{H}$ is said to vanish at infinity if for all $\epsilon>0$ there exists a compact subset $C$ of $\widehat{H}$ such that $|f(\chi)|<\epsilon$ for all $\chi \notin C$.

Proposition 3.1. Let $V=\mathbb{R}^{d}, d \geq 2$, and $C$ be a compact connected Lie subgroup of $G L(V)$ whose action has no nonzero fixed point in $V$. Let $\lambda \in P(V)$ be a measure invariant under the $C$-action. Let $A$ be an affine subspace of $V$ such that $\lambda(A)>0$. Then $A$ is a vector subspace of $V$.

Proof. Let $U$ be a minimal affine subspace contained in $A$ with $\lambda(U)>0$. By the minimality condition for any $g \in C$ either $g U=U$ or $\lambda(U \cap g U)=0$. Let $C^{\prime}=\{g \in C \mid g U=U\}$; then $C^{\prime}$ is a closed subgroup of $C$. Suppose that $C^{\prime}$ is a proper closed subgroup. As $C$ is a connected Lie group it follows that $C / C^{\prime}$ is uncountable. We can therefore find an uncountable subset $E$ of $C$ consisting of elements belonging to distinct cosets of $C^{\prime}$, so for any distinct $g, g^{\prime}$ in $E$ we have 
$g^{-1} g^{\prime} \notin C^{\prime}$. Then $\lambda\left(g U \cap g^{\prime} U\right)=\lambda\left(U \cap g^{-1} g^{\prime} U\right)=0$ for any distinct $g, g^{\prime}$ in $E$, which means that $\{g U\}_{g \in E}$ are pairwise essentially disjoint. But this is not possible since $\lambda$ is $C$-invariant, $\lambda(U)>0$ and $E$ is uncountable. Therefore $C^{\prime}=C$, which means that $U$ is $C$-invariant. Now we consider $V$ equipped with a $C$-invariant Hilbert norm. Then the point of $U$ closest to the origin would be a fixed point for the $C$-action. Since by hypothesis there is no nonzero fixed point, the point must be the origin. Therefore $U$, and in turn $A$, contains 0 . This shows that $A$ is a vector subspace.

Theorem 3.2. Let $V=\mathbb{R}^{d}, d \geq 2$, and $C$ be a compact connected Lie subgroup of $G L(V)$ whose action has no nonzero fixed point in $V$. Let $\lambda \in P(V)$ be a $C$ invariant measure on $V$, such that there is no proper vector subspace of positive $\lambda$-measure. Then $\lambda^{d}$ has a density on $V$ with respect to the Lebesgue measure; in particular, $\widehat{\lambda}$ vanishes at infinity.

Proof. For all $x \in V \backslash\{0\}, C x$ is an analytic submanifold of $V$ of positive dimension. For any $x \in V$ we denote by $T_{x}$ the tangent space to $C x$ at $x$; then $\operatorname{dim} T_{x}>0$ for all $x \neq 0$. We shall first show that for almost all $\left(x_{1}, \ldots, x_{d}\right)$ in $V^{d}$, with respect to the measure $\lambda^{\otimes d}$ (the $d$ th Cartesian power of $\lambda$ ), $T_{x_{1}}+\cdots+T_{x_{d}}=V$. We observe that, if $x_{1}, x_{2}, \cdots, x_{n}$ is a finite sequence in $V$ such that $T_{x_{k+1}}$ is not contained in $\sum_{i=1}^{k} T_{x_{i}}$ for $k=1, \ldots, n-1$, then $k \mapsto \operatorname{dim}\left(\sum_{i=1}^{k} T_{x_{i}}\right)$ is strictly increasing, hence $n \leq d$. For $\omega=\left(x_{1}, \cdots, x_{d+1}\right) \in V^{d+1}$ we denote by $n(\omega)$ the least $k \leq d$ such that $T_{x_{k+1}} \subset \sum_{i=1}^{k} T_{x_{i}}$, and by $p(\omega)$ the dimension of $\sum_{i=1}^{n(\omega)} T_{x_{i}}$; then $n(\omega) \leq p(\omega) \leq d$. Let $p$ be the essential infimum of the function $p(\omega)$ over $V^{d+1}$ with respect to the measure $\lambda^{\otimes(d+1)}$. If we show that $p=d$ it would follow that $\sum_{i=1}^{d} T_{x_{i}}=V, \lambda^{\otimes d}$-a.e..

Suppose $p<d$. Then there exist $x_{1}, \ldots, x_{k}$, for some $k \leq p$ such that $\sum_{i=1}^{k} T_{x_{i}}$ is a $p$-dimensional subspace and $T_{x} \subset \sum_{i=1}^{k} T_{x_{i}}$ for all $x$ in a set of positive $\lambda$ measure. Let $W=\sum_{i=1}^{k} T_{x_{i}}$ and $S=\left\{x \in V \mid T_{x} \subset W\right\}$. Then we have $\lambda(S)>0$. For $x \in V$ let $m_{\bar{x}}$ denote the normalised $C$-invariant measure on $C x$, let $\bar{V}$ be the space of $C$-orbits and $\bar{\lambda}$ the projection of $\lambda$ on $\bar{V}$. We decompose $\lambda$ into conditional measures over the quotient space. Since $\lambda$ is $C$-invariant the conditional measures are $m_{\bar{x}}$ for $\bar{\lambda}$-almost all $\bar{x} \in \bar{V}$. Thus $\lambda=\int m_{\bar{x}} d \bar{\lambda}(\bar{x})$. Let $S^{\prime}=\left\{x \in S \mid m_{\bar{x}}(S)>0\right\}$; then $\lambda\left(S^{\prime}\right)=\lambda(S)>0$. Consider $x \in S^{\prime}$. Since the map $y \mapsto T_{y}$ is analytic over $y \in C x$, and $T_{y} \subset W$ for $y$ in a set of positive $m_{\bar{x}}$-measure it follows that $T_{y} \subset W$ for all $y \in C x$. Now if $\{\gamma(t)\}$ is a differentiable curve on $C x$ and $l$ is a linear form on $V$ vanishing on $W$, then the condition that $T_{y} \subset W$ for all $y \in C x$ implies that the derivative of $l \circ \gamma$ is zero. This shows that $C x$ is contained in $x+W$. Let $U$ be the subspace spanned by $\left\{k x-k^{\prime} x \mid k, k^{\prime} \in C\right\}$. Then $U$ is a $C$-invariant subspace of $W$ and also $x+U$ is $C$-invariant. We consider $V$ equipped with a $C$-invariant Hilbert norm. Then $x+U$ has a unique point 
of minimum norm, and hence the point must be fixed under the $C$-action. Since by hypothesis there are no nonzero fixed points we get that $0 \in x+U$, which means that $x \in U \subset W$. Thus we have shown that $S^{\prime}$ is contained in $W$. Hence $\lambda(W) \geq \lambda\left(S^{\prime}\right)>0$. Since $W$ is of dimension $p<d$ and by hypothesis there is no proper subspace of positive $\lambda$-measure this is a contradiction. This implies that $p=d$, and hence $\sum_{i=1}^{k} T_{x_{i}}=V, \lambda^{\otimes d}$ - a.e..

We now consider the map $\varphi: V^{d} \rightarrow V$ given by $\varphi(\omega)=x_{1}+\cdots+x_{d}$ for $\omega=$ $\left(x_{1}, \cdots, x_{d}\right) \in V^{d}$, and the Borel set $E=\left\{\omega=\left(x_{1}, \cdots, x_{d}\right) \in V^{d} \mid \sum_{i=1}^{d} T_{x_{i}}=V\right\}$ which satisfies $\lambda^{\otimes d}(E)=1$. For $\omega \in E$, the differential of $\varphi$ at $\omega$ is surjective, and hence for some neighbourhood $\Delta$ of $\omega$ in $C x_{1} \times \cdots \times C x_{d}, \varphi(\Delta)$ is an open subset of $V$; also the push forward by $\varphi$ of the restriction to $\Delta$ of $m_{\bar{x}_{1}} \otimes \cdots \otimes m_{\bar{x}_{d}}$ has a density with respect to Lebesgue measure on $V$. Since this property is valid $\lambda^{\otimes d}$ - a.e., and $\lambda^{d}=\int \varphi\left(m_{\bar{x}_{1}} \otimes \cdots \otimes m_{\bar{x}_{d}}\right) d \lambda^{\otimes d}(\omega)$, we get that $\lambda^{d}$ has also a density with respect to Lebesgue measure on $V$. The last assertion in the statement of the theorem follows from the Riemann-Lebesgue Lemma.

\section{Asymptotics of measures under shear transfor- mations}

In this section we apply Proposition 3.1 to prove certain general results about asymptotics of measures under the action of sequences from a special class of automorphisms; see [4] for other results on the theme.

Let $L$ be a locally compact second countable group. Suppose that $L$ has a torus $T$ contained in its center, and a closed normal subgroup $R$ such that $L / R$ is a vector group. Let $W=L / R$ and $\theta: L \rightarrow W$ be the quotient homomorphism. For any $\alpha \in \operatorname{Hom}(W, T)$ we get an automorphism $\tilde{\alpha}$ of $L$ defined by $\tilde{\alpha}(g)=g \alpha(g R)$ for all $g \in G$; such an automorphism is called a shear automorphism.

We consider below Hom $(W, T)$ as a vector space; it will be convenient to use the additive notation, with the symbol " + " for the group operation in $\operatorname{Hom}(W, T)$. For any $\mu \in P(L)$ let $X(\mu)=\{\alpha \in \operatorname{Hom}(W, T) \mid \tilde{\alpha}(\mu)=\mu\}$.

Lemma 4.1. Let the notation be as above. Let $\mu \in P(L)$. Suppose that every affine subspace of $W$ of positive $\theta(\mu)$-measure is a vector subspace. Then $X(\mu)$ is a vector subspace of Hom $(W, T)$.

Proof. Let $\alpha \in X(\mu)$ be a nontrivial element and let $\left\{\alpha_{t}\right\}$ be the unique line in Hom $(W, T)$ containing $\alpha$. Let $\left\{\mu_{w}\right\}_{w \in W}$ be the conditional measures of $\mu$ over the fibration $\theta: L \rightarrow W$. We note that for any $\alpha \in X(\mu)$ and $w \in W, \theta^{-1}(w)$ is $\alpha$-invariant. It follows that for $\theta(\mu)$-almost all $w, \mu_{w}$ is invariant under translation by $\alpha(w)$. The set of $w$ in $W$ for which the subgroup generated by $\alpha(w)$ is not 
dense in the closed subgroup generated by $\left\{\alpha_{t}(w) \mid t \in \mathbb{R}\right\}$ is a countable union of affine subspaces which are not vector subspaces; to see this note that for such a $w$ there exists a character $\chi$ on $T$ such that $\chi(\alpha(w))$ is a nontrivial element of finite order. Since affine subspaces other than vector subspaces are of $\theta(\mu)$-measure 0 this implies that for $\theta(\mu)$-almost all $w, \mu_{w}$ is invariant under translation by $\left\{\alpha_{t}(w)\right\}$. Hence $\mu$ is $\left\{\tilde{\alpha}_{t}\right\}$-invariant, so $\alpha_{t} \in X(\mu)$ for all $t \in \mathbb{R}$. The conclusion shows that $X(\mu)$ is connected, so being a closed subgroup of Hom $(W, T)$ it is a vector subspace.

A measure $\lambda$ on $V=\mathbb{R}^{d}, d \geq 2$ is said to be pure if every vector subspace with positive $\lambda$-measure contains the subspace spanned by supp $\lambda$; the latter can be a proper subspace. It can be seen that every $\lambda \in P(V)$ can be expressed uniquely as a countable sum $\lambda=\sum_{i \in I} \lambda_{i}$ where $\left\{\lambda_{i}\right\}_{i \in I}$ is a family, finite or countably infinite, of pure measures on $V$, and for any distinct $i, j \in I$ the subspaces spanned by $\operatorname{supp} \lambda_{i}$ and $\operatorname{supp} \lambda_{j}$ are distinct; we call $\lambda_{i}$ 's the pure components of $\lambda$. For ready reference later we note the following.

Lemma 4.2. Let $\lambda \in P\left(\mathbb{R}^{n}\right), n \geq 2$, and let $\lambda=\sum_{i \in I} \lambda_{i}$ be the decomposition of $\lambda$ into pure components, where $I$ is an indexing set. For all $i$ let $W_{i}$ be the subspace spanned by $\operatorname{supp} \lambda_{i}$. Then the following statements hold:

i) If $\lambda$ is invariant under the action of a connected subgroup $C$ of $G L(n, \mathbb{R})$ then each $\lambda_{i}$ is $C$-invariant.

ii) If for all $i$ there exist $d_{i} \in \mathbb{N}$ such that $\lambda_{i}^{d_{i}}$ has a density with respect to the Lebesgue measure on $W_{i}$, then every affine subspace of $\mathbb{R}^{n}$ with positive $\lambda$-measure is a vector subspace.

Proof. i) Let $C$ be a connected subgroup of $G L(n, \mathbb{R})$ such that $\lambda$ is $C$-invariant. By the uniqueness of the collection of pure components $\lambda_{i}, i \in I, C$ must permute the components. For any $i \in I$ the subgroup $C_{i}$ of elements of $C$ leaving $\lambda_{i}$ invariant is a closed subgroup, and as $I$ is countable we get that $C / C_{i}$ is countable; since $C$ is connected this implies that $C=C_{i}$, so $\lambda_{i}$ is $C$-invariant.

ii) Let $A$ be an affine subspace such that $\lambda(A)>0$. Let $i \in I$ be such that $\lambda_{i}(A)>0$. Let $B=\left\{\sum_{j=1}^{d_{i}} v_{j} \mid v_{j} \in A\right\}=\left\{d_{i} v \mid v \in A\right\}$. Then $\lambda_{i}^{d_{i}}(B) \geq \lambda_{i}(A)^{d_{i}}>$ 0 . Since $\lambda_{i}^{d_{i}}$ is supported on $W_{i}$ and has a density on $W_{i}$ this implies $B$ contains $W_{i}$. In particular $0 \in B$, and since $B=d_{i} A$ we get that $0 \in A$. Therefore $A$ is a vector subspace.

Theorem 4.3. Let $\mu$ be a finite measure on L. Let $\theta(\mu)=\sum_{i \in I} \lambda_{i}$ be the decomposition of $\theta(\mu)$ into pure components and for all $i$ let $W_{i}$ be the subspace spanned by $\operatorname{supp} \lambda_{i}$. Suppose that for all $i$ there exists $d_{i}$ such that $\lambda_{i}^{d_{i}}$ has a density with respect to the Lebesgue measure on $W_{i}$. Let $\left\{\alpha_{j}\right\}$ be a sequence in Hom $(W, T)$ such that $\left\{\tilde{\alpha}_{j}(\mu)\right\}$ converges to a measure of the form $\tau(\mu)$, for some continuous auto- 
morphism $\tau$ of $L$ fixing $T$ pointwise. Then in the quotient space Hom $(W, T) / X(\mu)$ the sequence $\left\{\alpha_{j} X(\mu)\right\}$ is relatively compact.

Proof. The measure $\mu$ can be expressed uniquely as $\mu=\sum_{i \in I} \mu_{i}$, where $\mu_{i} \in P(L)$, $i \in I$, are such that $\theta\left(\mu_{i}\right)=\lambda_{i}$ for all $i$. Then $X(\mu)=\cap X\left(\mu_{i}\right)$. By Lemmas 4.1 and 4.2 the condition in the hypothesis implies that each $X\left(\mu_{i}\right)$ is a vector subspace of Hom $(W, T)$. Therefore to show that $\left\{\alpha_{j}+X(\mu)\right\}$ is relatively compact in Hom $(W, T) / X(\mu)$ it suffices to show that $\left\{\alpha_{j}+X\left(\mu_{i}\right)\right\}$ is relatively compact for all $i$. In other words in proving the theorem we may assume that $\theta(\mu)$ is a pure measure.

Let $E$ be the subset of $\widehat{T}$ consisting of all $\chi \in \widehat{T}$ such that $\left\{\chi \circ \alpha_{j}\right\}$ is a bounded subset of $\widehat{T}$. Then $E$ is a subgroup of $\widehat{T}$. Also, the defining condition for $E$ readily implies that $\widehat{T} / E$ has no nontrivial element of finite order. Let $S$ be the annihilator of $E$ in $T$. Then $S$ is a compact subgroup, and the preceding observation implies that $S$ is connected, namely a torus. Let $S^{\prime}$ be a subtorus of $T$ such that $T=S^{\prime} S$, a direct product. Then each $\alpha_{j}$ can be decomposed as $\beta_{j}+\gamma_{j}$ canonically, with $\beta_{j} \in \operatorname{Hom}\left(W, S^{\prime}\right)$, and $\gamma_{j} \in \operatorname{Hom}(W, S)$, with Hom $\left(W, S^{\prime}\right)$ and Hom $(W, S)$ viewed as subgroups of Hom $(W, T)$ canonically.

We note that $\left\{\beta_{j}\right\}$ is relatively compact in Hom $\left(W, S^{\prime}\right)$. To see this it suffices to know that $\left\{\chi \circ \beta_{j}\right\}$ is bounded for all characters $\chi$ on $S^{\prime}$. Since the latter, viewed as characters on $T$, belong to $E$ the desired property is immediate from the definition of $E$.

We now consider a Borel section $s$ of $L / T$ into $L$ such that $\Lambda:=s(L / T)$ is locally compact, and identify $L$ and $\Lambda \times T$ as Borel spaces. For $\varphi \in C_{c}(L / T)$ and $\chi \in \widehat{T}$, we denote by $\varphi \otimes \chi$ the function on $L$ defined by $\varphi \otimes \chi(x t)=\varphi(x) \chi(t)$, for all $x \in \Lambda$ and $t \in T$. The set of such functions is separating in $P(L)$, as is easily verified using disintegration of measures on $L$ with respect to the projections of $L$ on $L / T$. Hence, we can test weak convergence of measures on $L$, using these functions. We disintegrate $\mu$ as $\mu=\int \delta_{x} \otimes \mu_{x} d \bar{\mu}(x)$ where $\mu_{x} \in P(T)$ for all $x \in \Lambda$ and $\bar{\mu} \in P(L / T)$ is the projection of $\mu$ on $L / T$.

Viewing elements of Hom $(W, T)$ also as functions on $L / T$ via the canonical projection of $L / T$ on to $L / R$, for the measures $\tilde{\alpha}_{j}(\mu)$ we see that

$$
\widetilde{\alpha}_{j}(\mu)(\varphi \otimes \chi)=\int \varphi(x) \chi(t) \chi\left(\alpha_{j}(x)\right) d \mu_{x}(t) d \bar{\mu}(x)=\int \varphi(x) \widehat{\mu}_{x}(\chi)\left(\chi \circ \alpha_{j}\right)(x) d \bar{\mu}(x)
$$

for all $j$. We denote by $\mu_{\varphi}^{\chi}$ the projection on $W=L / R$ of the measure with density $\varphi(x) \widehat{\mu}_{x}(\chi)$ with respect to $\bar{\mu}$. Then the value of the above integral is $\widehat{\mu}_{\varphi}^{\chi}\left(\chi \circ \alpha_{j}\right)$. Since by the condition in the hypothesis a power $\theta(\mu)^{d}$ of $\theta(\mu)$ has a density with respect to the Lebesgue measure on $W$, the same holds for the measure $\mu_{\varphi}^{\chi}$, and hence $\widehat{\mu}_{\varphi}^{\chi}$ vanishes at infinity on $\widehat{W}$. Then, for $\chi \notin E$, and in particular for any 
nontrivial character $\chi$ from $\widehat{S} \subset \widehat{T},\left\{\chi \circ \alpha_{j}\right\}$ is unbounded on $\widehat{W}$, and hence the preceding conclusion together with the fact that $\tilde{\alpha}_{j}(\mu) \rightarrow \tau(\mu)$ implies that $\tau(\mu)(\varphi \otimes \chi)=0$. This means that the measure $\tau(\mu)$ is translation-invariant under the action of elements of $S$. Since $\tau$ is a continuous automorphism that pointwise fixes $T$, and in particular $S$, it follows that $\mu$ is translation-invariant under the $S$-action; thus $\mu_{x}$ is $S$-invariant for almost all $x \in L / T$. Since for all $j$ the image of $\gamma_{j}$ is contained in $S$, this shows that $\mu$ is invariant under $\widetilde{\gamma}_{j}$ for all $j$. Hence $\gamma_{j} \in X(\mu)$ for all $j$. Since $\alpha_{j}=\beta_{j} \gamma_{j}$ for all $j$ and $\left\{\beta_{j}\right\}$ is relatively compact this shows that $\left\{\alpha_{j}+X(\mu)\right\}$ is relatively compact.

\section{Some properties of roots of measures}

Given a Lie group $G$, a closed subgroup $H$ of $G$ and a subset $\Lambda$ of $P(G)$ we say that $\Lambda / H$ is relatively compact, if for every sequence $\left\{\lambda_{i}\right\}$ in $\Lambda$ there exists a sequence $\left\{h_{i}\right\}$ in $H$ such that $\left\{\lambda_{i} h_{i}\right\}$ is relatively compact in $P(G)$.

Theorem 5.1. Let $G$ be a Lie group (not necessarily connected) and suppose that there exists a continuous homomorphism $r: G \rightarrow \widetilde{G}$ onto an almost algebraic group $\widetilde{G}$, such that ker $r$ is a discrete central subgroup of $G$. Let $\sigma \in P(G)$ and let $\Psi$ be a set of roots of $\sigma$ in $P(G)$. Let $M$ be the smallest closed normal $G(\Psi)$ invariant subgroup of $Z^{0}(\sigma)$ such that $Z^{0}(\sigma) / M$ is a vector group and the induced $G(\Psi)$-action on $Z^{0}(\sigma) / M$ is trivial. Then $\Psi / M$ is relatively compact.

Proof. Let $\nu=r(\sigma)$. We first prove that $r\left(Z^{0}(\sigma)\right)=Z^{0}(\nu)$ : Let $C=r^{-1}(Z(\nu))^{0}$. For any $g \in G(\sigma),\left\{g z g^{-1} z^{-1} \mid z \in C\right\}$ is a connected subset contained in ker $r$, and as the latter is discrete it consists only of the identity element. Therefore $C$ is contained in $Z^{0}(\sigma)$. Also, since $\operatorname{ker} r$ is discrete, $r(C)=Z^{0}(\nu)$. Thus we have $Z^{0}(\nu) \subseteq r\left(Z^{0}(\sigma)\right)$. The other way inclusion is easy to see.

We next show that $\Psi / Z(\sigma)$ is relatively compact. Let $\left\{\lambda_{i}\right\}$ be a sequence in $\Psi$. Then $\lambda_{i}$ are factors of $\sigma$ and by a standard argument involved in the factor compactness theorems (see [6], [7], [4]) there exists a sequence $\left\{x_{i}\right\}$ in $N(\sigma)$ such that $\left\{\lambda_{i} x_{i}\right\}$ and $\left\{x_{i}^{-1} \sigma x_{i}\right\}$ are relatively compact in $P(G)$. Then $\left\{r\left(x_{i}\right)^{-1} \nu r\left(x_{i}\right)\right\}$ is relatively compact in $P(\widetilde{G})$. Since $\widetilde{G}$ is almost algebraic this implies that $\left\{r\left(x_{i}\right) Z^{0}(\nu)\right\}$ is relatively compact in $\widetilde{N}(\nu) / Z^{0}(\nu)$ (see [7]). Since $r\left(Z^{0}(\sigma)\right)=Z^{0}(\nu)$ this yields that $\left\{x_{i} Z^{0}(\sigma)(\operatorname{ker} r)\right\}$ is relatively compact in $N(\sigma) / Z^{0}(\sigma)(\operatorname{ker} r)$, and as $Z^{0}(\sigma)(\operatorname{ker} r)$ is contained in $Z(\sigma)$ it follows that $\left\{x_{i} Z(\sigma)\right\}$ is relatively compact in $N(\sigma) / Z(\sigma)$. As $\left\{\lambda_{i} x_{i}\right\}$ is relatively compact this implies in turn that there exists a sequence $\left\{z_{i}\right\}$ in $Z(\sigma)$ such that $\left\{\lambda_{i} z_{i}\right\}$ is relatively compact. Therefore $\Psi / Z(\sigma)$ is relatively compact.

Now let $\Phi=r(\Psi)$. We note that $\widetilde{G}(\Phi)$ normalises $Z^{0}(\nu)$ and as they are almost algebraic subgroups the product $\widetilde{G}(\Phi) Z^{0}(\nu)$ is also almost algebraic and in 
particular a closed subgroup of $\widetilde{G}$. Let $H=r^{-1}\left(\widetilde{G}(\Phi) Z^{0}(\nu)\right)$. Let $\pi: H \rightarrow H / M$ be the quotient homomorphism. Now, $\pi(Z(\sigma))$ is a closed subgroup and as $\Psi / Z(\sigma)$ is relatively compact we get that $\pi(\Psi) / \pi(Z(\sigma))$ is relatively compact.

Let $Z^{\prime}(\sigma)=Z^{0}(\sigma)(\operatorname{ker} r)$. Then $Z^{\prime}(\sigma)$ is a closed subgroup and $r\left(Z^{\prime}(\sigma)\right)=$ $Z^{0}(\nu)$. Since $Z^{0}(\nu)$ is of finite index in $Z(\nu)$ this implies that $Z(\sigma) / Z^{\prime}(\sigma)$ is finite. Hence we get that $\pi(\Psi) / \pi\left(Z^{\prime}(\sigma)\right)$ is relatively compact. From the definition of $M$ it follows that the conjugation action of $G(\Psi)$ on $\pi\left(Z^{0}(\sigma)\right)$ is trivial. The subgroup of $\widetilde{G}$ consisting of elements whose conjugation action on $\pi\left(Z^{0}(\sigma)\right)$ is trivial is an almost algebraic subgroup of $\widetilde{G}$. Since $\operatorname{ker} r$ is central and $\widetilde{G}(\Phi)$ is the smallest almost algebraic group containing $r(G(\Psi))$ it follows that the conjugation action of $r^{-1}(\widetilde{G}(\Phi))$ on $\pi\left(Z^{0}(\sigma)\right)$ is trivial. Since $Z^{0}(\sigma) / M$ is abelian this shows that $\pi\left(Z^{0}(\sigma)\right)$ is contained in the center of $H / M$, and hence so is $\pi\left(Z^{\prime}(\sigma)\right)$. Together with the fact that $\pi(\Psi) / \pi\left(Z^{\prime}(\sigma)\right)$ is relatively compact, by Proposition 3.4 of [8] we get that $\{\pi(\Psi)\}$ is relatively compact. Since $\Psi / Z(\sigma)$ is relatively compact and ker $\pi=M$ this shows that $\Psi / M$ is relatively compact; for a sequence $\left\{\lambda_{i}\right\}$ in $\Psi$ if $\left\{z_{i}\right\}$ is a sequence in $Z(\sigma)$ such that $\left\{\lambda_{i} z_{i}\right\}$ is relatively compact then we get that $\left\{\pi\left(z_{i}\right)\right\}$ is relatively compact and so $\left\{z_{i}\right\}$ can be replaced by a sequence in ker $\pi$. This proves the theorem.

As before let $Q$ be the smallest closed normal subgroup of $\widetilde{G}(\nu)$ such that $\widetilde{G}(\nu) / Q$ is a vector group. We note that $Q$ is the subgroup of $\widetilde{G}(\nu)$ containing $[\widetilde{G}(\nu), \widetilde{G}(\nu)]$, such that $Q /[\widetilde{G}(\nu), \widetilde{G}(\nu)]$ is the maximal compact subgroup of $\widetilde{G}(\nu) /[\widetilde{G}(\nu), \widetilde{G}(\nu)]$. Hence $Q$ is an almost algebraic subgroup of $\widetilde{G}$. Moreover, since $\widetilde{G}(\nu) / Q$ is a vector group, and in particular has no finite subgroup, it follows that $Q$ is Zariski closed in $\widetilde{G}(\nu)$. Therefore $\widetilde{G}(\nu) / Q$ is an almost algebraic group. Let $\widetilde{V}=\widetilde{G}(\nu) / Q$, equipped with its structure as an algebraic group. The characteristic property of $Q$ as a subgroup of $\widetilde{G}(\nu)$ shows also that $Q$ is normal in $\widetilde{N}(\nu)$. Let $\eta: \widetilde{N}(\nu) \rightarrow \widetilde{N}(\nu) / Q$ be the canonical quotient homomorphism; we have $\eta(\widetilde{G}(\nu))=\widetilde{V}$. For $g \in \widetilde{N}(\nu)$ let $c_{g}: \widetilde{V} \rightarrow \widetilde{V}$ be the transformation induced by the conjugation action of $g$ on $\widetilde{G}(\nu)$. Let $\widetilde{N}_{1}(\nu)$ be the subgroup of $\widetilde{N}(\nu)$ consisting of all elements $g$ such that the measure $\eta(\nu)$ is invariant under the action of $c_{g}$.

Remark 5.2. Given a Lie group $G$ (or more generally a locally compact group) and $\mu \in P(G)$, if $G(\mu)$ is abelian and $\lambda$ is a root of $\mu$ supported on $x G(\mu)$, with $x \in N(\mu)$, then $\mu$ is invariant under the conjugation action of $x$ on $G(\mu)$ (see [11, Lemma 2.2).

Proposition 5.3. Let the notation be as above. Then we have the following;

i) $\left\{c_{g} \mid g \in \widetilde{N}_{1}^{0}(\nu)\right\}$ is a compact subgroup of $G L(\widetilde{V})$;

ii) if $\Phi$ is a set of roots of $\nu$ in $P(\widetilde{G})$ then $\left\{c_{g} \mid g \in \widetilde{G}(\Phi)\right\}$ is compact and preserves $\eta(\nu)$. 
Proof. i) This follows from Corollary 2.5 of [2], since $\left\{c_{g} \mid g \in \widetilde{N}(\nu)\right\}$ is an almost algebraic subgroup of $G L(V)$ and $\widetilde{G}(\eta(\nu))=\widetilde{V}$.

ii) For all $\rho \in \Phi, \eta(\rho)$ is a root of $\eta(\nu) \in P(\widetilde{V})$ and as $\widetilde{V}$ is abelian, by Remark $5.2 \operatorname{supp} \rho$ is contained in $\widetilde{N}_{1}(\nu)$. The compactness of $\left\{c_{g} \mid g \in \widetilde{N}_{1}^{0}(\nu)\right\}$ implies in particular that $\widetilde{N}_{1}(\nu)$ is an almost algebraic subgroup. Therefore $\widetilde{G}(\Phi)$ is contained in $\widetilde{N}_{1}(\nu)$. Also, since $\widetilde{G}(\Phi)$ is an almost algebraic subgroup $\left\{c_{g} \mid g \in\right.$ $\widetilde{G}(\Phi)\}$ is closed. Hence by (i) it is compact, and it preserves $\eta(\nu)$, since $\widetilde{G}(\Phi)$ is contained in $\widetilde{N}_{1}(\nu)$.

\section{$6 \quad$ Embedding of infinitely divisible measures}

Our next objective is to prove the following theorem, from which Theorem 1.3 can be deduced.

For any finite measure $\lambda$ on a Lie group $G$ we denote by $I(\lambda)$ the subgroup $\{g \in G \mid g \lambda=\lambda g\}$ and by $J(\lambda)$ the subgroup $\{g \in G \mid g \lambda=\lambda g=\lambda\}$. We note that $I(\lambda)$ and $J(\lambda)$ are closed subgroups and $J(\lambda)$ is normal in $I(\lambda)$.

Theorem 6.1. Let $G, T$ and $q: G \rightarrow G / T$ be as in Theorem 1.3. Let $\mu \in P(G)$, $\sigma=q(\mu)$, and suppose that $\sigma$ has no nontrivial idempotent factor in $P(G / T)$. Let $\Psi$ be a root cluster of $\mu$, and let $M$ be the smallest closed normal $G(\Psi)$-invariant subgroup of $Z^{0}(\sigma)$ such that $Z^{0}(\sigma) / M$ is a vector group and the induced $G(\Psi)$ action on $Z^{0}(\sigma) / M$ is trivial. Suppose that, in $G / T, G(q(\Psi))$ normalizes $q(I(\mu)) \cap$ $M$. Then there exists a rational embedding $\left\{\mu_{r}\right\}_{r \in \mathbb{Q}^{+}}$of $\mu$ such that $\mu_{r} \in \Psi$ for all $r \in \mathbb{Q}^{+}$.

Apart from the results of the previous sections, for the proof of Theorem 6.1 we need certain general results. We begin by recalling these.

Firstly we recall the following result from [5], extending analogous results proved in [9] under some restrictions on the subgroup $N$ as in the statement of the theorem below. Let $H$ be a Lie group and $\sigma \in P(H)$. We recall (from [5]) that a root $\rho$ of $\sigma$ is said to be compatible with a Lie subgroup $N$ of $N(\sigma)$ if $\rho \in P(x G(\sigma))$ for some $x \in N(\sigma)$ which normalises $N$. The following is Corollary 2.4 from [5], with a co-finite subset of $\mathbb{N}$ in the place of $\Sigma$ there.

Theorem 6.2. Let $H$ be a Lie group. Let $\sigma \in P(H)$ and for each $k$ let $\rho_{k}$ be a $k$ ! th root of $\sigma$. For all $n \in \mathbb{N}$ let $S_{n}=\left\{\rho_{k}^{k ! / n !} \mid k \geq n\right\}$. Let $N$ be a closed compactly generated nilpotent subgroup of $Z(\sigma)$. Suppose that all $\rho_{k}$ are compatible with $N$ and that $S_{n} / N$ is relatively compact for all large $n$. Then there exist sequences $\left\{k_{j}\right\}$ in $\mathbb{N},\left\{z_{j}\right\}$ in $N^{0}$ and $n_{0} \in \mathbb{N}$ such that $k_{j} \rightarrow \infty$ as $j \rightarrow \infty$ and $\left\{z_{j} \rho_{k_{j}}^{k_{j} ! / n !} z_{j}^{-1} \mid j \in \mathbb{N}, k_{j} ! \geq n !\right\}$ is relatively compact for all $n \geq n_{0}$. 
We need also the following result from [18]. It may be noted that the condition in the hypothesis of Theorem 6.1 is connected with the use of this theorem.

Theorem 6.3. Let $G$ be a Lie group and $\sigma \in P(G)$. Let $\lambda_{j} \in P(G)$ be a $n_{j}$ th root of $\sigma$, where $n_{j} \rightarrow \infty$ as $j \rightarrow \infty$. Let $\left\{x_{j}\right\}$ be a sequence in $Z(\sigma)$ such that $\left\{\lambda_{j} x_{j}\right\}$ converges to a measure $\lambda \in P(G)$. Then there exists $x \in I(\sigma)$ such that the support of $\lambda$ is contained in $x J(\sigma)$.

Proof. Each $\lambda_{j} x_{j}$ is a factor of $\sigma$ and hence it follows that the limit $\lambda$ is a factor of $\sigma$. Also, for any $k \in \mathbb{N},\left(\lambda_{j} x_{j}\right)^{k}$ can be expressed as $\lambda_{j}^{k} y_{j}^{(k)}$, with $y_{j}^{(k)} \in Z(\sigma)$ (see [9], Proposition 2.2 for an idea of the proof). Hence $\left(\lambda_{j} x_{j}\right)^{k}$ is a factor of $\sigma$ for all $j$ such that $n_{j} \geq k$. As $\left(\lambda_{j} x_{j}\right)^{k} \rightarrow \lambda^{k}$ as $j \rightarrow \infty$ and $n_{j} \rightarrow \infty$, we get that $\lambda^{k}$ is a factor of $\sigma$ for all $k$. The theorem now follows from Theorem 2.4 of [18].

To prove the general case of Theorem 6.1 we will need also the following theorem, which enables to reduce to a situation where $Z^{0}(\sigma)$ contains a cocompact simply connected nilpotent normal subgroup. We postpone proving it, until $\S 7$, and go over to the proof of Theorem 6.1; we note that for certain Lie groups, such as the groups involved in Corollary 1.5, the conclusion of Theorem 6.4 is readily seen to hold.

Theorem 6.4. Let $\widetilde{G}$ be an almost algebraic group and $\nu \in P(\widetilde{G})$. Let $\Phi$ be a set of roots of $\nu$. Suppose that $\widetilde{G}(\Phi) / \widetilde{G}(\nu)$ is connected. Let $K$ be the smallest almost algebraic normal subgroup of $Z^{0}(\nu)$ such that $Z^{0}(\nu) / K$ is a vector group. Then there exists an almost algebraic subgroup $\widetilde{H}$ of $\widetilde{G}(\Phi)$ such that the following conditions are satisfied:

i) for all $\rho \in \Phi$ there exists $z \in K$ such that $z \rho z^{-1} \in P(\widetilde{H})$, and

ii) if $U$ is the unipotent radical of $\widetilde{G}^{0}(\Phi)$ and $P=\widetilde{G}(\nu) U$, then $\widetilde{H}$ contains $P$ and the quotient $\left(Z^{0}(\nu) \cap \widetilde{H}\right) /\left(Z^{0}(\nu) \cap P\right)$ is compact; furthermore $Z^{0}(\nu) \cap \widetilde{H}$ contains a simply connected nilpotent cocompact normal almost algebraic subgroup.

We now begin the proof of Theorem 6.1. Let the notation be as in the hypothesis. Also, let $\nu=p(\mu)$. Let $\Psi$ be a root cluster of $\mu$ in $P(G)$. The subgroup $\widetilde{G}(p(\Psi))$ (see $\S 2$ for definition) being an almost algebraic subgroup has only finitely many connected components; let $c$ be the number of the components. Now, let $\Psi^{\prime}=\left\{\rho^{c} \mid \rho, \rho^{c} \in \Psi\right\}$. It is straightforward to see that $\Psi^{\prime}$ is also a root cluster of $\mu$ contained in $\Psi$. Furthermore $\widetilde{G}\left(p\left(\Psi^{\prime}\right)\right) / \widetilde{G}(\nu)$ is connected. Therefore, in proving Theorem 6.1, replacing $\Psi$ by $\Psi^{\prime}$ and modifying the notation we may assume that $\widetilde{G}(p(\Psi)) / \widetilde{G}(\nu)$ is connected.

The conditions of Theorem 6.4 are satisfied for $\widetilde{G}, \nu$ as above and $\Phi=p(\Psi)$. Let $\widetilde{H}$ be an almost algebraic subgroup of $\widetilde{G}(\Phi)$ for which the assertions as in that theorem are satisfied. Now let $G^{\prime}=p^{-1}(\widetilde{H}) / T$, and let $q: p^{-1}(\widetilde{H}) \rightarrow G^{\prime}$ 
and $r: G^{\prime} \rightarrow \widetilde{H}$ be the quotient homomorphisms. We note that $r$ is a covering homomorphism onto the almost algebraic group $\widetilde{H}$. Now let $\Psi^{*}=\Psi \cap P\left(p^{-1}(\widetilde{H})\right)$. We note that $\Psi^{*}$ is a root cluster for $\mu$, which we now view as a measure on $p^{-1}(\widetilde{H})$ : given $n \in \mathbb{N}$ there exists $\rho \in \Psi$ such that $\rho^{n}=\mu$ and by condition (i) in the conclusion of Theorem 6.4 there exists $x \in p^{-1}(K)$ such that $x \rho x^{-1} \in P\left(p^{-1}(\widetilde{H})\right)$; since $p^{-1}(K) \subset Z(\mu)$ and $\Psi$ is a root cluster we get that $x \rho x^{-1} \in \Psi$, showing that $\Psi^{*}$ contains a $n$th root of $\mu$, for any $n$; the other conditions as in the definition of a root cluster are straightforward to verify. Replacing $\Psi$ by $\Psi^{*}$ if necessary and modifying the notation, in proving Theorem 6.1 we may without loss of generality assume that $\Psi$ is contained in $p^{-1}(\widetilde{H})$.

Now let $M$ be the subgroup as in the hypothesis of Theorem 6.1. Then by Theorem $5.1 q(\Psi) / M$ is relatively compact. By assertion (ii) of Theorem $6.4 Z^{0}(\nu) \cap \widetilde{H}$ admits a cocompact almost algebraic simply connected nilpotent normal subgroup. We note that $r(M)$ is an almost algebraic subgroup of $Z^{0}(\nu) \cap \widetilde{H}$ and hence we get that $r(M)$ contains a cocompact almost algebraic simply connected nilpotent normal subgroup. Since such a subgroup is necessarily unique, it follows that it is normalised by $\widetilde{G}(\Phi)$. Since $r\left(Z^{0}(q(\mu))\right)=Z^{0}(\nu) \cap \widetilde{H}$ this implies that $M$ contains a cocompact simply connected nilpotent normal subgroup, invariant under the action of $\widetilde{G}(\Phi)$; we shall denote the subgroup, which is unique, by $N$. Then $q(\Psi) / N$ is relatively compact.

Towards the proof of the Theorem 6.1 we shall now first prove the following.

Proposition 6.5. Let the notation be as above. Also, for all $k \in \mathbb{N}$ let $\rho_{k}$ be a $k$ ! th root of $\mu$ contained in $\Psi$. For all $n \in \mathbb{N}$ let $S_{n}=\left\{q\left(\rho_{k}\right)^{k ! / n} \mid k \geq n\right\} \subseteq P\left(G^{\prime}\right)$. Let $N^{\prime}=N \cap q(I(\mu))$ Then $S_{n} / N^{\prime}$ is relatively compact for all large $n$.

Proof. Let $R$ be the subgroup of $p^{-1}(\widetilde{G}(\nu))$ consisting of all $g$ such that $\psi_{p(z)}(p(g))=$ $e$, the identity element, for all $z \in q^{-1}(M)$. By Lemma $2.1 R$ contains $p^{-1}(Q)$. Therefore $p^{-1}(\widetilde{G}(\nu)) / R$ is a quotient of $\widetilde{G}(\nu) / Q$ and hence a vector group. Let $W=p^{-1}(\widetilde{G}(\nu)) / R$, equipped with the induced vector space structure. Also, $p(R) / Q$ is invariant under the action of $\widetilde{G}(\Phi)$ on $\widetilde{G}(\nu) / Q$. Moreover by Corollary 2.4 every fixed point of the $\widetilde{G}(\Phi)$-action on $\widetilde{G}(\nu) / Q$ is contained in $p(R) / Q$. Since the $\widetilde{G}(\Phi)$-action on $\widetilde{G}(\nu) / Q$ is through a compact group (see Proposition 5.3) this further implies that the induced $\widetilde{G}(\Phi)$-action on $W$ has no nonzero fixed point.

Let $\theta: p^{-1}(\widetilde{G}(\nu)) \rightarrow W$ be the quotient homomorphism. We write $\mu$ as $\mu_{0}+\mu^{\prime}$, where $\mu_{0}$ is supported on $R$ and $\mu^{\prime}(R)=0$.

Now let $\Delta=\{(k, n) \mid k, n \in \mathbb{N}, k \geq n\}$. For any $\delta=(k, n) \in \Delta$ let $\theta_{\delta}=$ $q\left(\rho_{k}\right)^{k ! / n}$. As $q(\Psi) / N$ is relatively compact we get that there exists a family $\left\{x_{\delta}\right\}_{\delta \in \Delta}$ in $N$ such that $\left\{\theta_{\delta} x_{\delta}\right\}$ is relatively compact. Moreover for a subset $E$ of $\Delta$ and a closed normal subgroup $H$ of $N,\left\{\theta_{\delta} \mid \delta \in E\right\} / H$ is relatively compact if and 
only if $\left\{x_{\delta} H\right\}_{\delta \in E}$ is relatively compact in $N / H$. We note that $\psi_{z}$ is trivial for all $z \in[N, N]$, and hence it follows that $[N, N]$ is contained in $q(I(\mu))$. Therefore $N \cap q(I(\mu))$ is a normal subgroup of $N$. To prove the proposition it therefore suffices to show that $\left\{x_{(k, n)} \mid k \geq n\right\} /(N \cap q(I(\mu)))$ is relatively compact for all large $n$. By the definition of $R, \psi_{p(z)}(p(g))=e$ for all $g \in R$ and $z \in q^{-1}(M)$, and in particular for all $z \in q^{-1}(N)$. Since the support of $\mu_{0}$ is contained in $R$, this implies that $q\left(I\left(\mu_{0}\right)\right)$ contains $N$. Hence $N \cap q(I(\mu))=N \cap q\left(I\left(\mu^{\prime}\right)\right)=N^{\prime}$, say.

Suppose, if possible that $\left\{x_{(k, n)} N^{\prime} \mid k \geq n\right\}$ is not relatively compact for infinitely many $n$. We can then find sequences $\left\{k_{j}\right\}$ and $\left\{n_{j}\right\}$ in $\mathbb{N}$, such that, $k_{j} \geq n_{j}$, $n_{j} \rightarrow \infty$ and $\left\{x_{\left(k_{j}, n_{j}\right)} N^{\prime}\right\}$ has no convergent subsequence in $N / N^{\prime}$. For all $j \in \mathbb{N}$ let $\theta_{j}=q\left(\rho_{k_{j}}^{k_{j} ! / n_{j}}\right)$ and $x_{j}=x_{\left(k_{j}, n_{j}\right)}$. Then $\left\{\theta_{j} x_{j}\right\}$ is relatively compact. Let $\lambda \in P\left(G^{\prime}\right)$ be a limit point of $\left\{\theta_{j} x_{j}\right\}$. By Theorem 6.3 there exists $x \in I(q(\mu))$ such that $\operatorname{supp} \lambda$ is contained in $x J(q(\mu))$ (notation as in Theorem 6.3). The normalised Haar measure of $J(q(\mu))$ is an idempotent factor of $q(\mu)$, and since by hypothesis $q(\mu)$ has no nontrivial idempotent factor we see that $J(q(\mu))$ is trivial. Hence $\lambda=\delta_{x}$ with $x \in I(q(\mu))$.

For every $j$ let $\phi_{j}$ be a $n_{j}$ th root of $\mu$ such that $q\left(\phi_{j}\right)=\theta_{j}$. Let $y_{j} \in G$ be such that $q\left(y_{j}\right)=x_{j}$. Then $\left\{\phi_{j} y_{j}\right\}$ has a limit point, say $\alpha \in P(G)$ such that $q(\alpha)=\lambda=\delta_{x}$. Let $y \in G$ be such that $q(y)=x$. Then $\alpha=y \beta$ for some $\beta \in P(T)$.

For all $j$ we have the identity $\mu=\left(\phi_{j} y_{j}\right)\left(y_{j}^{-1} \phi_{j}^{k_{j}-1}\right)$. Since $\left\{\phi_{j} y_{j}\right\}$ is relatively compact it follows also that $\left\{y_{j}^{-1} \phi_{j}^{k_{j}-1}\right\}$ is relatively compact (cf. [17], Chapter III, Theorem 2.1) and hence has a subsequence which converges, to say $\gamma \in P(G)$. Then passing to limit along such a subsequence we have $\mu=y \beta \gamma$. Also $\left\{y_{j}^{-1} \mu y_{j}\right\}=$ $\left\{\left(y_{j}^{-1} \phi_{j}^{k_{j}-1}\right)\left(\phi_{j} y_{j}\right)\right\}$ converges along the subsequence to $\gamma(y \beta)=\gamma \beta y=\beta \gamma y=$ $y^{-1} \mu y$; note that since $\beta \in P(T), y \beta=\beta y$ and $\gamma \beta=\beta \gamma$. Thus $y^{-1} \mu y$ is a limit of a subsequence of $\left\{y_{j}^{-1} \mu y_{j}\right\}$. It follows also that $\left\{y_{j}^{-1} \mu^{\prime} y_{j}\right\}$ has a subsequence converging to $y^{-1} \mu^{\prime} y$.

Now let $L=p^{-1}(\widetilde{G}(\nu))$. We apply Theorem 4.3 to this $L$, with $W$ as above, and $\mu^{\prime}$ in the place of $\mu$ there. Let $\lambda=\theta\left(\mu^{\prime}\right)$. We recall that the $\widetilde{G}(\Phi)$-action on $W$ has no nonzero fixed point, and it preserves $\lambda$ (see Proposition 5.3). Then by Theorem [3.2, the pure components $\lambda_{i}$ of $\lambda$ are such that $\lambda_{i}^{d_{i}}$ have a density on the subspace spanned by $\operatorname{supp} \lambda_{i}$, for some $d_{i}$, and hence the condition in the hypothesis of Theorem 4.3 is satisfied. We note also for any $z \in q^{-1}(N)$ and $g \in L$ we have $z^{-1} g z=g \alpha_{z}(\theta(g))$, where $\alpha_{z}: W \rightarrow T$ is the map given by $\alpha_{z}(\theta(g))=g^{-1} z^{-1} g z=\psi_{z^{-1}}(p(g))$ (in the notation as before) for all $g \in L$. Since $\left\{y_{j}^{-1} \mu^{\prime} y_{j}\right\}$ has a subsequence converging to $y^{-1} \mu^{\prime} y$, in view of these observations Theorem 4.3 implies that the sequence $\left\{x_{j} N^{\prime}\right\}$ has a convergent subsequence in $N / N^{\prime}$. As this contradicts the choice of $\left\{x_{j}\right\}$, we get that $S_{n} / N^{\prime}$ is relatively compact for all large $n \in \mathbb{N}$. This proves the proposition. 
Completion of the proof of Theorem 6.1, We follow the notation as above. By Proposition 6.5 $S_{n} / N^{\prime}$ is relatively compact for all large $n$. Recall that by hypothesis $q(I(\mu)) \cap M$ is normalized by $\widetilde{G}(\Phi)$. Also, as seen above $N$ is normalized by $\widetilde{G}(\Phi)$. Hence $N^{\prime}=N \cap(q(I(\mu)) \cap M)$ is normalized by $\widetilde{G}(\Phi)$. This shows that all $q\left(\rho_{k}\right), k \in \mathbb{N}$ are compatible with $N^{\prime}$. Hence by Theorems 6.2 there exist sequences $\left\{k_{j}\right\}$ in $\mathbb{N},\left\{z_{j}\right\}$ in $N^{\prime}$ and $n_{0} \in \mathbb{N}$ such that $k_{j} \rightarrow \infty$ as $j \rightarrow \infty$, and $\left\{z_{j} q\left(\rho_{k_{j}}\right)^{k_{j} ! / n !} z_{j}^{-1} \mid j \in \mathbb{N}, k_{j} ! \geq n !\right\}$ is relatively compact for all $n \geq n_{0}$. For all $j$ let $\zeta_{j} \in q^{-1}\left(z_{j}\right)$. Since the conjugation action of $q^{-1}\left(N^{\prime}\right)$ leaves $\mu$ invariant, each $\zeta_{j} \rho_{k_{j}}^{k_{j} ! / n !} \zeta_{j}^{-1}$ is a root of $\mu$. For all $n \geq n_{0}$, since $\left\{q\left(\zeta_{j} \rho_{k_{j}}^{k_{j} ! / n !} \zeta_{j}^{-1}\right)\right\}=$ $\left\{z_{j} q\left(\rho_{k_{j}}\right)^{k_{j} ! / n !} z_{j}^{-1}\right\}$ is relatively compact and the kernel of $q$ is compact it follows that $\left\{\zeta_{j} \rho_{k_{j}}^{k_{j} ! / n !} \zeta_{j}^{-1}\right\}$ is relatively compact. Following a standard argument with limits we can now find $k$ ! th roots $\mu_{1 / k !}, k \geq 2$, such that $\left(\mu_{1 / k !}\right)^{k}=\mu_{1 /(k-1) \text { ! for all }}$ $k$. We now define for $r=p / q$, where $p, q \in \mathbb{N}, \mu_{r}$ to be $\mu_{1 / q !}^{p(q-1) !}$; in view of the preceding conditions it follows that $\mu_{r}$ defined in this way is indeed independent of the representation of $r$ as $p / q$. It is also straightforward to see that $\left\{\mu_{r}\right\}$ is a rational embedding of $\mu$ contained in $\Psi$. This proves the first statement in the theorem. As noted before the later statements are well-known consequences of the first one. This completes the proof of the theorem (assuming the validity of Theorem 6.4).

\section{Measures on almost algebraic groups}

We now prove Theorem 6.4 on "reducing" an almost algebraic group so that $Z(\nu)$ contains a simply connected nilpotent cocompact normal subgroup. The proof is along the lines of the reduction theorem in [8] and [9], the crucial additional point being that the conjugating elements are chosen from the subgroup $K$, rather than the whole of $Z(\nu)$.

Proof of Theorem 6.4 Let $U$ and $P$ be as in (ii) in the statement of the theorem. As $\widetilde{G}(\nu)$ and $U$ are normal almost algebraic subgroups of $\widetilde{G}(\Phi)$, so is $P$. As $U$ is the unipotent radical of $\widetilde{G}^{0}(\Phi), \widetilde{G}(\Phi) / P$ is a reductive almost algebraic group. Also, since $\widetilde{G}(\Phi) / \widetilde{G}(\nu)$ is connected, so is $\widetilde{G}(\Phi) / P$. Now let $Z^{\prime}=(\widetilde{G}(\Phi) \cap Z(\nu))^{0} P$, which is an almost algebraic subgroup of $\widetilde{G}(\Phi)$. Then $Z^{\prime} / P$ is a connected almost algebraic normal subgroup of $\widetilde{G}(\Phi) / P$ and hence there exists an almost algebraic normal subgroup $R$ of $\widetilde{G}(\Phi)$ containing $P$ and such that $\widetilde{G}(\Phi) / P$ is the almost direct product of $R / P$ and $Z^{\prime} / P$, namely, $\widetilde{G}(\Phi)=R Z^{\prime}$ and $\left(R \cap Z^{\prime}\right) / P$ is finite. Now let $\widetilde{H}$ be a closed subgroup of $\widetilde{G}(\Phi)$ containing $R$ and such that $\widetilde{H} / R$ is a maximal compact subgroup of $\widetilde{G}(\Phi) / R$. Let $K^{\prime}=K \cap Z^{\prime}$. Then $K^{\prime}$ is an almost algebraic normal subgroup of $Z^{\prime}$ and $Z^{\prime} / K^{\prime}$ is a vector group. We note that $R K^{\prime}$ 
is an almost algebraic normal subgroup of $\widetilde{G}(\Phi)$ such that $\widetilde{G}(\Phi) / R K^{\prime}$ is a vector group, and hence in particular $\widetilde{H}$ is contained in $R K^{\prime}$. Clearly $\widetilde{H}$ is a maximal compact subgroup of $R K^{\prime}$.

Let $\rho \in \Phi$ and $x \in \widetilde{G}(\Phi)$ be such that $\rho \in P(x \widetilde{G}(\nu))$. As $x \widetilde{G}(\nu)$ is of finite order in $\widetilde{G}(\Phi) / \widetilde{G}(\nu)$, so is $x R$ in $\widetilde{G}(\Phi) / R$. By conjugacy of maximal compact subgroup we therefore get that $x R$ is conjugate in $K^{\prime} R / R$ to an element of $\widetilde{H} / R$. Therefore there exists a $z \in K^{\prime}$ such that $z x z^{-1} \in \widetilde{H}$. Hence $z \rho z^{-1} \in P(\widetilde{H})$. This proves (i).

Now, $\left.\left(Z^{0}(\nu)\right) \cap \widetilde{H}\right) P$ is an almost algebraic and hence a closed subgroup, and $\left.\left(Z^{0}(\nu)\right) \cap \widetilde{H}\right) P / P$ is topologically isomorphic to $\left(Z^{0}(\nu) \cap \widetilde{H}\right) /\left(Z^{0}(\nu) \cap P\right)$. Therefore to prove (ii) it suffices to show that $\left(\widetilde{H} \cap Z^{0}(\nu)\right) P / P$ is compact. Recall that $\widetilde{G}(\Phi) / P$ is an almost direct product of $Z^{0}(\nu) P / P$ and $R / P$. Also $\widetilde{H}$ contains $R$ and $\widetilde{H} / R$ is compact. The preceding observation therefore implies that $\left(\widetilde{H} \cap Z^{0}(\nu)\right) P / P$ is compact, as sought to be shown.

Now let $W$ be the unipotent radical of $Z(\nu) \cap P$. We show that $(Z(\nu) \cap P)^{0}$ is contained in $(Z(\nu) \cap \widetilde{G}(\nu)) W$. As $W$ is the unipotent radical of $Z(\nu) \cap P$ by Levi decomposition there exists a reductive almost algebraic subgroup $L$ such that $(Z(\nu) \cap P)^{0}=L W$. Since $U$ is contained in the unipotent radical of $P^{0}$, we get that there exists $u \in U$ such that $u L u^{-1}$ is contained in $\widetilde{G}^{0}(\nu)$. But $U$ is contained in $\widetilde{N}(\nu)$, so this means that $L$ is contained in $\widetilde{G}(\nu)$. This proves the claim as above. Now let $A$ be the maximal almost algebraic vector subgroup of the abelian almost algebraic group $\widetilde{G}(\nu) \cap Z(\nu)$; in particular $\widetilde{G}(\nu) \cap Z(\nu) / A$ is compact. Then $A W$ is a normal almost algebraic subgroup of $Z(\nu) \cap \widetilde{H}$ and since $A$ centralizes $W, A W$ is a simply connected nilpotent Lie group. Also, since $Z^{0}(\nu) \cap P$ is cocompact in $Z^{0}(\nu) \cap \widetilde{H}$ the above observation shows that $\left(Z^{0}(\nu) \cap \widetilde{H}\right) / A W$ is compact. This proves the theorem.

\section{Concluding results}

In this section we shall deduce the results stated in the introduction. To relate Theorem 1.3 to Theorem 6.1 we need the following proposition.

Proposition 8.1. Let the notation $G, \widetilde{G}, p, q$ and $T$ be as in Theorem 1.3. Let $\mu \in P(G), \sigma=q(\mu) \in P(G / T)$ and $\nu=p(\mu) \in P(\widetilde{G})$. Let $\Psi$ be a root cluster of $\mu$ such that $\widetilde{G}(p(\Psi)) / \widetilde{G}(\nu)$ is connected. Let $M$ be the subgroup of $Z^{0}(\sigma)$ as in Theorem 6.1. Then $q(I(\mu)) \cap M$ is normalized by $G(q(\Psi))$.

Proof. Let $R$ be the subgroup of $p^{-1}(\tilde{G}(\nu))$ consisting of all $g$ in $G$ such that $\psi_{p(z)}(p(g))=e$ for all $z \in q^{-1}(M)$. From Remark 2.2 we see that $R$ is a closed normal subgroup of $p^{-1}(\tilde{G}(\nu))$ and $p^{-1}(\tilde{G}(\nu)) / R$ is a vector group, a quotient of 
$\widetilde{V}$, which we shall denote by $W$. Also, $R$ is invariant under the conjugation action of $G(\Psi)$ and by Corollary 2.4 the induced $G(\Psi)$-action on $p^{-1}(\tilde{G}(\nu)) / R$ has no nonzero fixed point. Let $\Phi=p(\Psi)$ and $G_{1}=p^{-1}(\widetilde{G}(\Phi))$; we note that $G_{1}$ contains $p^{-1}(\tilde{G}(\nu))$ and $R$ as normal subgroups. Let $\theta: G_{1} \rightarrow G_{1} / R$ be the quotient homomorphism; then $\theta\left(p^{-1}(\tilde{G}(\nu))\right)=W$.

We write $\mu$ as $\mu_{0}+\mu^{\prime}$ where $\mu_{0} \in P(R)$ and $\mu^{\prime}(R)=0$. As $q^{-1}(M)$ centralizes $R$ it follows that $I(\mu) \cap q^{-1}(M)=I\left(\mu^{\prime}\right) \cap q^{-1}(M)$. Now we express $\mu^{\prime}$ as $\mu^{\prime}=\sum_{i} a_{i} \mu_{i}$, with $i$ running over a suitable countable indexing set, $a_{i}$ 's positive real numbers such that $\sum_{i} a_{i}=1$, and $\mu_{i}$ are probability measures such that $a_{i} \theta\left(\mu_{i}\right)$ are the pure components of $\theta(\mu)$; (see $\S 3$ ). Such a decomposition is unique and in particular this implies that $I(\mu) \cap q^{-1}(M)=\cap_{i}\left(I\left(\mu_{i}\right) \cap q^{-1}(M)\right)$. For each $i$ let $W_{i}$ be the subspace of $W$ spanned by $\theta\left(\mu_{i}\right)$, and let $d_{i}=\operatorname{dim} W_{i}$. By Proposition 5.3, $\theta(\mu)$ is invariant under the induced action of $\widetilde{G}(\Phi)$ on $W$. The action of $\widetilde{G}(\nu)$ on $W$ is trivial, and hence the $\widetilde{G}(\Phi)$-action involved is through the quotient group $\widetilde{G}(\Phi) / \widetilde{G}(\nu)$. As the latter is a connected group, by Lemma 4.2 we get that $a_{i} \theta\left(\mu_{i}\right)$, which are the pure components of $\theta(\mu)$, are $\widetilde{G}(\Phi)$-invariant for all $i \in I$. In particular each $W_{i}$ is also $\widetilde{G}(\Phi)$-invariant, and in view of the invariance of the measure the action on $W_{i}$ is via a compact subgroup of $G L\left(W_{i}\right)$. Furthermore since the $\widetilde{G}(\Phi)$-action on $W$ has no nonzero fixed point and by hypothesis $\widetilde{G}(\Phi) / \widetilde{G}(\nu)$ is connected, by Theorem 3.2 we get that $\theta\left(\mu_{i}\right)^{d_{i}}$ has a density in $W_{i}$.

Now consider the partition of $G$ into cosets of $T$ and let $\left\{\mu_{\xi}\right\}_{\xi \in G / T}$ be a system of conditional measures for $\mu$ with respect to the partition. It is easy to see that there exists a sequence of pairwise disjoint Borel subsets $\left\{B_{i}\right\}$ of $G / T$ such that $q\left(\mu_{i}\right)\left(B_{i}\right)=1$. Therefore the system of conditional measures as above can also be chosen so that it is a system of conditional measures for each $\mu_{i}$, over the quotient measure $q\left(\mu_{i}\right)$, for each index $i$. For $g \in q^{-1}(M)$, each fibre $\xi=x T$, $x \in G$, is invariant under the conjugation action of $g$ and the action on $\xi$ is given by translation by the element $g_{\xi}=g x g^{-1} x^{-1} \in T$, which is independent of the choice of $x$ in $\xi$. Hence for $g \in I(\mu) \cap q^{-1}(M)$, for $\sigma$-almost all $\xi, \mu_{\xi}$ is invariant under the translation action of $g_{\xi}$. For $\xi \in G / T$ let $S_{\xi}$ be the smallest closed subgroup of $T$ containing $\left\{g_{\xi} \mid g \in I(\mu) \cap q^{-1}(M)\right\}$. Then for $\sigma$-almost all $\xi, \mu_{\xi}$ is invariant under $S_{\xi}$. We shall show that $S_{\xi}$ is constant $q\left(\mu_{i}\right)$-a.e.. Let $\mathcal{C}$ denote the family of all closed subgroups of $T$. We recall that $C$ is countable. For $C \in \mathcal{C}$, let $I_{C}=\left\{\xi \in G / T \mid S_{\xi} \subset C\right\}$. Then $I_{C}$ is a closed subgroup of $G / T$. We have $\sigma\left(\cup_{C \in \mathcal{C}} I_{C}\right)=1$, and hence for every index $i, q\left(\mu_{i}\right)\left(\cup_{C \in \mathcal{C}} I_{C}\right)=1$.

Now consider any index $i$. By the above observation there exists $C \in \mathcal{C}$ such that $q\left(\mu_{i}\right)\left(I_{C}\right)>0$. Then $\theta\left(\mu_{i}\right)\left(\theta\left(q^{-1}\left(I_{C}\right)\right)\right)>0$, and since $\theta\left(\mu_{i}\right)^{d_{i}}$ has a density when viewed as a measure on $W_{i}$, this implies that the closed subgroup $\theta\left(q-1\left(I_{C}\right) \cap W_{i}\right.$ has positive Lebesgue measure in $W_{i}$, hence it equals $W_{i}$ and so $\theta\left(\mu_{i}\right)\left(\theta\left(q^{-1}\left(I_{C}\right)\right)=1\right.$. On the other hand $q^{-1}\left(I_{C}\right)$ is a subgroup containing 
$R=\operatorname{ker} \theta$, and hence $\theta\left(\mu_{i}\right)\left(\theta\left(q^{-1}\left(I_{C}\right)\right)\right)=\mu_{i}\left(q^{-1}\left(I_{C}\right)\right)=q\left(\mu_{i}\right)\left(I_{C}\right)$, so we have $q\left(\mu_{i}\right)\left(I_{C}\right)=1$. Let $\mathcal{C}^{\prime}$ be the family of all $C \in \mathcal{C}$ such that $q\left(\mu_{i}\right)\left(I_{C}\right)=1$; by our observations above $\mathcal{C}^{\prime}$ is nonempty. It can be seen from dimension and cardinality considerations that every nonempty collection of subgroups from $\mathcal{C}$ has a minimal element. Let $C_{0}$ be the minimal element in $\mathcal{C}^{\prime}$. Then $q\left(\mu_{i}\right)\left(I_{C_{0}}\right)=1$ and $q\left(\mu_{i}\right)\left(I_{C}\right)=0$ for all $C \in \mathcal{C}$ such that $C \subset C_{0}$ and $C \neq C_{0}$. It follows that for $q\left(\mu_{i}\right)$-almost all $\xi, S_{\xi}=C_{0}$. Let $E=\left\{x \in G \mid S_{x T}=C_{0}\right\}$; then we have $\mu_{i}(E)=1$. Since for any $\xi \in G / T$ and $\zeta \in R / T$ we have $g_{\xi \zeta}=g_{\xi} g_{\zeta}=g_{\xi}$ we get that $E R=E$. Also, as noted above $\theta\left(\mu_{i}\right)$ is invariant under the action of $\widetilde{G}(\Phi)$ and hence in particular that of $G(\Psi)$. Therefore for any $y \in G(\Psi)$, we have $\mu_{i}\left(y E y^{-1}\right)=\mu_{i}\left(y E y^{-1} R\right)=\theta\left(\mu_{i}\right)\left(\theta\left(y E y^{-1}\right)\right)=\theta\left(\mu_{i}\right)(\theta(E))=\mu_{i}(E)=1$.

Now let $g \in I(\mu) \cap q^{-1}(M)$ and $y \in G(\Psi)$ be given. In particular $g \in I\left(\mu_{i}\right)$. Also, $q^{-1}(M)$ being $G(\psi)$-invariant, we get that $y g y^{-1} \in q^{-1}(M)$ and its action on the fibre $\xi=x T$ is given by

$$
\left(y g y^{-1}\right) x\left(y g y^{-1}\right)^{-1} x^{-1}=y g\left(y^{-1} x y\right) g^{-1}\left(y^{-1} x y\right)^{-1} y^{-1}=g\left(y^{-1} x y\right) g^{-1}\left(y^{-1} x y\right)^{-1},
$$

the last equality being a consequence of the element being contained in the center; thus the action is by translation by $g_{y(\xi)}$, where $y(\xi)=\left(y^{-1} x y\right) T$. It follows that if $\xi \in G / T$ is such that both $\xi$ and $y(\xi)$ are contained in $E$, then $\mu_{\xi}$ is invariant under the action of $y g y^{-1}$. Since $\mu_{i}(E)=\mu_{i}\left(y E y^{-1}\right)=1$ this indeed holds for $q\left(\mu_{i}\right)$-almost all $\xi$, which shows that $\mu_{i}$ is invariant under the conjugation action of $y g y^{-1}$, namely $y g y^{-1} \in I\left(\mu_{i}\right)$. Since this holds for all $i$ it follows that $y g y^{-1} \in I(\mu)$. This proves the proposition.

Proof of Theorem 1.3. As in the proof of Theorem 6.1, replacing $\Psi$ by a root cluster $\Psi^{*}$ contained in it (consisting of certain fixed powers) we may assume that such that $\widetilde{G}(p(\Psi)) / \widetilde{G}(p(\mu))$ is connected. When this holds, by Proposition 8.1 the condition as in Theorem 6.1 is satisfied and hence it follows that there exists a rational embedding $\left\{\mu_{r}\right\}_{r \in \mathbb{Q}^{+}}$of $\mu$ such that $\mu_{r} \in \Psi$ for all $r \in \mathbb{Q}^{+}$. This proves the theorem.

Proof of Corollary 1.4 Let $T$ be the maximal torus in $N$. Then there exists a representation $\rho: G \rightarrow G L(n, \mathbb{R})$, for some $n \in \mathbb{N}$, such that ker $\rho$ is contained in the center of $G$ and $(\operatorname{ker} \rho)^{0}=T$ (cf. [16]). Now, $[\rho(G), \rho(G)]$ is an almost algebraic subgroup (cf. [1]) and also $\rho(N)$ is an algebraic subgroup (cf. [19]), so we get that $[\rho(G), \rho(G)] \rho(N)$ is almost algebraic. Let $G^{\prime}$ be the closed subgroup of $G$ containing $[G, G] N$ such that $G^{\prime} / \overline{[G, G] N}$ is the maximal compact subgroup of $G / \overline{[G, G] N}$. Then $G^{\prime}$ is a closed connected subgroup of $G$ such that $G / G^{\prime}$ is a vector group. The latter implies in particular that $G^{\prime}$ contains $\operatorname{ker} \rho$ and hence $\rho\left(G^{\prime}\right)$ is a closed subgroup of $\rho(G)$. From the choice of $G^{\prime}$ we now get that $\rho\left(G^{\prime}\right) /[\rho(G), \rho(G)] \rho(N)$ is compact, and since $[\rho(G), \rho(G)] \rho(N)$ is almost algebraic, this implies furthermore 
that $\rho\left(G^{\prime}\right)$ is algebraic. We can therefore apply Theorem 1.3 to $G^{\prime}$, in place of $G$ there. Now let $\mu \in P(N)$ and suppose that it is infinitely divisible in $P(G)$. Then $\mu \in P\left(G^{\prime}\right)$. Also, since $G / G^{\prime}$ has no elements of finite order, all roots of $\mu$ are supported on $G^{\prime}$ and so $\mu$ is infinitely divisible on $G^{\prime}$. Let $q: G^{\prime} \rightarrow G^{\prime} / T$ be the quotient homomorphism. As $T$ is the maximal torus in $N, N / T$ has no nontrivial compact subgroup. Since any idempotent factor is the Haar measure of a compact subgroup (see [13]) this shows that $q(\nu)$ has no nontrivial idempotent factor. Hence by Theorem $1.3 \mu$ is embeddable in $P\left(G^{\prime}\right)$, and hence also in $P(G)$.

For the proof of Corollary 1.5 we recall some known results. Let $G$ be a Lie group. A $\mu \in P(G)$ is said to be strongly root compact if the set $\left\{\rho^{k} \mid \rho^{n}=\mu, 1 \leq\right.$ $k \leq n\}$ is relatively compact in $P(G)$, and it is said to be factor compact if its set of (two-sided) factors, namely $\lambda \in P(G)$ for which there exists $\nu \in P(G)$ such that $\mu=\lambda \nu=\nu \lambda$, is compact. A factor compact measure is strongly root compact. A strongly root compact measure which is infinitely divisible is embeddable (see [13, [15]).

Proposition 8.2. Let $V=\mathbb{R}^{2}$ and let $G$ be the semidirect product of $S L(2, \mathbb{R})$ and $V$ with respect to the natural linear action. Let $\mu \in P(G)$ be such that $\mu \notin P(V)$. Then there exists a closed subgroup $G^{\prime}$ containing $G(\mu)$ such that the following conditions are satisfied: (i) if $\rho \in P(G)$ and $\rho^{2 n}=\mu$ for some $n \in \mathbb{N}$, then $\rho^{2} \in P\left(G^{\prime}\right)$, and (ii) $\mu$ is strongly root compact in $G^{\prime}$.

Proof. Let $\eta: G \rightarrow S L(2, \mathbb{R})$ be the quotient homomorphism, and let $\nu=\eta(\mu)$. Let $A$ be the subgroup of $S L(2, \mathbb{R})$ consisting of all diagonal matrices with positive entries, and let $N$ be the subgroup consisting of all upper triangular unipotent matrices. From the structure of subgroups of $S L(2, \mathbb{R})$ we know that at least one of the following must hold: (a) $\widetilde{G}(\nu)$ contains a conjugate $A N$ in $S L(2, \mathbb{R})$; (b) $\widetilde{G}(\nu)$ is a nontrivial compact subgroup; or (c) $\widetilde{G}^{0}(\nu)$ is a conjugate of $A$ or $N$ in $S L(2, \mathbb{R})$; see [14 for instance, for an idea of the proof. If (a) or (b) holds then it can be seen that $Z(\mu)$ is a compact subgroup of $G$. As $G$ is an algebraic group this implies that $\mu$ is factor compact (cf. [7]). Therefore it is strongly root compact, and the assertion in the proposition holds for $G^{\prime}=G$.

To prove the assertion when Condition (c) holds we may, after applying a suitable conjugation, assume that $\widetilde{G}^{0}(\nu)=A$ or $N$. Then $\widetilde{N}(\nu) / \widetilde{G}^{0}(\nu)$ has a normal subgroup $F$ containing 2 or 4 elements such that the corresponding quotient has no elements of finite order; if $\widetilde{G}^{0}(\nu)=A$ then $F$ has 4 elements and the quotient is trivial, while if $\widetilde{G}^{0}(\nu)=N$ then $F$ has 2 elements and the quotient is isomorphic to $\mathbb{R}$. Let $E$ be the subgroup of $\widetilde{N}(\nu)$ containing $\widetilde{G}^{0}(\nu)$ and such that $F=E / \widetilde{G}^{0}(\nu)$. Then $E^{0} \subset \widetilde{G}(\nu) \subset E$. Also, from the choice of $F$ we see that $\widetilde{N}(\nu) / E$ has no elements of finite order, and therefore it follows that all roots of $\nu$ are supported on $E$. Correspondingly, for $\mu$ we have $\eta^{-1}\left(E^{0}\right) \subset \widetilde{G}(\mu) \subset \eta^{-1}(E)$ and all its roots 
are supported on $\eta^{-1}(E)$. If $\widetilde{G}(\nu)=N$ we set $G^{\prime}=\eta^{-1}(N)$. Since $F$ as above has only two elements in this case, it follows that $\rho^{2} \in P\left(G^{\prime}\right)$ for all $\rho$ such that $\rho^{2 n}=\mu$ for some $n \in \mathbb{N}$. Also, $N$ is a connected nilpotent Lie group and hence it follows that $\mu$ is strongly root compact (see [13], Chapter 3). Thus the assertion as in the proposition holds in this case.

Next suppose that $\widetilde{G}(\nu) \neq N$. Under this condition, if $V \cap G(\mu)$ is nonzero, then $Z(\mu)$ is compact and hence we are through as before. We may therefore assume that $V \cap G(\mu)=\{0\}$. Then $V \cap \tilde{N}(\mu)$ is also trivial, since if $v \in V$ normalizes $\widetilde{G}(\mu)$ then $v$ is a fixed point of the linear action of $\widetilde{G}(\nu)$, which can not hold for any nonzero vector when $\widetilde{G}(\nu) \neq N$. Hence the restriction of $\eta$ to $\widetilde{N}(\mu)$ is an isomorphism of $\widetilde{N}(\mu)$ onto $E$. It therefore suffices to prove the desired statement for $\mu \in P(E)$ with all roots contained $E$. If $\widetilde{G}(\mu)$ is nonabelian, which is possible only in the case $\widetilde{G}^{0}(\nu)=A$, then $Z(\mu)$ is compact and the assertion in the proposition holds as before. If $\widetilde{G}(\mu)$ is abelian, we choose $G^{\prime}$ to be the abelian subgroup of index 2 in $E$, and see that the requisite conditions are satisfied for this choice. This proves the proposition.

Proof of Corollary 1.5. Let $G$ be the semidirect product of $S L(2, \mathbb{R})$ and $N$, as in the hypothesis, and $\mu \in P(G)$ be infinitely divisible. Let $T$ be the maximal torus in $N$. We shall show that $\mu$ is embeddable, and that if infinite divisibility holds in $P(H)$, where $H$ is a closed connected subgroup containing $G(\mu)$, then an embedding may also be found in $H$; in this respect it suffices to consider $H$ containing $T$ since for any closed connected subgroup $H, H T$ is a direct product of $H$ and a subtorus of $T$, and embeddability on the latter implies embeddability on $H$. If $\mu \in P(N)$ then Corollary 1.4 implies that $\mu$ is embeddable; we note also that if $\mu \in P(H)$ for a closed connected subgroup $H$ of $G$, containing $T$, then $H \cap N$ is contained in the nilradical of $H$ and hence Corollary 1.4 may be applied to $H$ in place of $G$ to conclude that $\mu$ is embeddable in $P(H)$.

Now suppose that $\mu \notin P(N)$. Let $q: G \rightarrow G / T$ be the quotient homomorphisms. Then $q(\mu) \notin P(N / T)$. Hence by Proposition 8.2 we get that there exists a closed subgroup $G^{\prime}$ of $G / T$ containing $G(q(\mu))$ such that for $\rho \in P(G / T)$ we have $\rho^{2} \in P\left(G^{\prime}\right)$ whenever $\rho^{2 n}=q(\mu)$ for some $n \in \mathbb{N}$, and $q(\mu)$ is strongly root compact on $G^{\prime}$. Let $L=q^{-1}\left(G^{\prime}\right)$. Then $\mu \in P(L)$ and if $n \in \mathbb{N}$ and $\rho \in P(G)$ is a $2 n$th root of $\mu$ then $\rho^{2} \in P(L)$. This shows that $\mu$ is infinitely divisible in $P(L)$. On the other hand since $q(\mu)$ is strongly root compact on $G^{\prime}$, and the kernel of $q$ is a compact central subgroup it follows that $\mu$ is strongly root compact on $L$ (see [15]). Together with infinite divisibility in $P(L)$ this implies that $\mu$ is embeddable in $P(L)$ (see [13], Chapter 3, or [15]). Hence $\mu$ is embeddable in $P(G)$. Also if $H$ is a closed connected subgroup of $G$ containing $T$ and such that $\mu$ is infinitely divisible on $H$, we can choose $L=H \cap q^{-1}\left(G^{\prime}\right)$ and arguing as above conclude that $\mu$ is embeddable in $P(L) \subset P(H)$. This proves the Corollary. 
Proof of Theorem 1.6. Let $G$ be a connected Lie group and $\mu \in P(G)$ be an infinitely divisible measure such that $G(\mu) / \overline{[G(\mu), G(\mu)]}$ is compact. Let $H$ be the closed subgroup of $G$ containing $[G, G]$ and such that $H / \overline{[G, G]}$ is the maximal compact subgroup of the connected abelian Lie group $G / \overline{[G, G]}$. The condition on $\mu$ implies that $G(\mu)$ is contained in $H$. Furthermore, since $G / H$ is a vector group we get that all roots of $\mu$ are supported on $H$, so $\mu$ is infinitely divisible on $H$. Therefore in proving the theorem, by suitably replacing $G$ we may assume that $G / \overline{[G, G]}$ is compact. When this condition is satisfied $G$ admits a representation $p: G \rightarrow \widetilde{G}$ onto an almost algebraic group $\widetilde{G}$, such that ker $p$ is contained in the center of $G$ and $(\operatorname{ker} p)^{0}$ is compact; this may be seen by arguing as in the first part of the proof of Corollary 1.4, and noting that compact connected linear groups are algebraic. We then proceed as in the proof of Theorem 1.3. In view of the assumption that $G(\mu) / \overline{[G(\mu), G(\mu)]}$ is compact, the vector space $\widetilde{V}$ as in the earlier sections is trivial, and $\mu$ is in fact supported on the subgroup $R$ as in $\S 6$. For such a measure it may be seen from the proof of Theorem 6.1 that the condition that $q(\mu)$ has no idempotent factor is not involved in completing the proof of the conclusion that every root cluster of $\mu$ contains a rational embedding of $\mu$. Thus the argument shows that $\mu$ is embeddable in $P(G)$ under the conditions as above.

Acknowledgement: The first named author would like to thank the Institut de Recherche Mathématique de Rennes (IRMAR), Université de Rennes I, France for hospitality while some of this work was done.

\section{References}

[1] C. Chevalley, Theorie des Groupes de Lie, Hermann, Paris (1968).

[2] S.G. Dani, On ergodic quasi-invariant measures of group automorphisms, Israel J. Math. 43 (1982), 62 - 74.

[3] S.G. Dani, On automorphism groups of connected Lie groups, Manuscripta Math. 74 (1992), 445-452.

[4] S.G. Dani, Asymptotic behaviour of measures under automorphisms, Probability Measures on Groups: Recent Directions and Trends, 149-178, Tata Inst. Fund. Res., Mumbai, 2006.

[5] S.G. Dani, Convolution roots and embeddings of probability measures on locally compact groups, Indian J. of Pure and Applied Math.

[6] S.G. Dani and M. McCrudden, On the factor sets of measures and local tightness of convolution semigroups over Lie groups, J. Theoret. Probab. 1 (1988), $357-370$. 
[7] S.G. Dani and M. McCrudden, Factors, roots and embeddability of measures on Lie groups, Math. Z. 199 (1988), 369-385.

[8] S.G. Dani and M. McCrudden, Embeddability of infinitely divisible distributions on linear Lie groups, Invent. Math. 110 (1992), 237-261.

[9] S.G. Dani and M. McCrudden, Convolution roots and embeddings of probability measures on Lie groups, Adv. Math. 209 (2007), 198-211.

[10] S.G. Dani, M. McCrudden and S. Walker, On the embedding problem for infinitely divisible distributions on certain Lie groups with toral center, Math. Z. 245 (2003), 781-790; erratum: Math. Z. 252 (2006), 457-458.

[11] S.G. Dani and Klaus Schmidt, Affinely infinitely divisible distributions and the embedding problem, Math. Res. Lett. 9 (2002), 607-620.

[12] M. Eichler, and D. Zagier, The Theory of Jacobi forms, Progress in Mathematics 55, Birkhäuser, Boston, MA, 1985.

[13] H. Heyer, Probability Measures on Locally Compact Groups, Springer, BerlinNew York, 1977.

[14] M. McCrudden, Infinitely divisible probabilities on $S L(2, \mathbb{C})$ are continuously embedded, Math. Proc. Cambridge Philos. Soc. 92 (1982), 101-107.

[15] M. McCrudden, The embedding problem for probabilities on locally compact groups, Probability Measures on Groups: Recent Directions and Trends, 331363, Tata Inst. Fund. Res., Mumbai, 2006.

[16] N. Nahlus, Note on faithful representations and a local property of Lie groups, Proc. Amer. Math. Soc. 125 (1997), 2767-2769.

[17] K.R. Parthasarathy, Probability Measures on Metric Spaces, reprint of the 1967 original, AMS Chelsea Publishing, Providence, RI, 2005.

[18] Riddhi Shah, The central limit problem on locally compact groups, Israel J. Math. 110 (1999), 189-218.

[19] V.S. Varadarajan, Lie Groups, Lie Algebras, and Their Representations, Springer, New York, 1984 (reprint of the 1974 edition).

S.G. Dani: dani@math.tifr.res.in

Yves Guivarc'h: yves.guivarch@univ-rennes1.fr

Riddhi Shah: rshah@mail.jnu.ac.in 